\title{
Fuzzy Cognitive Maps in Business Analysis and Performance-Driven Change
}

\author{
George Xirogiannis and Michael Glykas
}

\begin{abstract}
Business process reengineering (BPR) has made a significant impact on managers and academics. Despite the rhetoric surrounding BPR, articulated mechanisms, which support reasoning on the effect of the redesign activities to the performance of the business model, are still emerging. This paper describes an attempt to build and operate such a reasoning mechanism as a novel supplement to performance-driven change (PDC) exercises. This new approach proposes the utilization of the fuzzy causal characteristics of fuzzy cognitive maps (FCMs) as the underlying methodology in order to generate a hierarchical and dynamic network of interconnected performance indicators. By using FCMs, the proposed mechanism aims at simulating the operational efficiency of complex process models with imprecise relationships to quantify the impact of performance-driven reengineering activities. This research also establishes generic maps that supplement the strategic planning and business analysis phases of typical redesign projects in order to implement the integration of hierarchical FCMs into PDC activities. Finally, this paper discusses experiments with the proposed mechanism and comments on its usability.
\end{abstract}

Index Terms-Business analysis, business process reengineering (BPR), fuzzy cognitive mapping, performance metrics.

\section{INTRODUCTION}

$\mathbf{T}$ HERE has been a significant change in management attitudes and practices in the last decade. This shift results from the understanding that enterprises should focus on meeting the customers' needs and aspirations, while engaging employees as efficiently as possible. With increasing competition in the global market, enterprises are seeking ways to improve internal capabilities and position themselves for survival in the future [16]. It is now common sense that successful enterprises should consist of fewer managerial layers, however, each layer should be entrusted with greater responsibilities for the daily business operations [45]. Moreover, enterprises should become team-oriented; the old rigid boundaries between human resources should be replaced with internal information networks, while the emphasis should be on achieving the overall goals of the enterprise.

Business process reengineering (BPR) is one general approach widely taken to implement internal changes. BPR focuses on innovation and creativity in redesigning processes in an effort to meet customers' needs and expectations. It can be defined as the fundamental rethinking of process models to

Manuscript received June 1, 2003; revised February 1, 2004. Review of this manuscript was arranged by Department Editor A. Marucheck.

The authors are with the Department of Financial and Management Engineering, University of the Aegean, 31, Chios 82 100, Greece (e-mail: g.xirogiannis@fme.aegean.gr; mglikas@aegean.gr).

Digital Object Identifier 10.1109/TEM.2004.830861 achieve dramatic improvements in critical performance indicators like cost, service quality, production speed, etc. Since BPR may involve dramatic internal changes, at some point almost everyone in the enterprise has to be involved. Hence, there is a need for monitoring the change initiatives in every organizational level of the enterprise. Furthermore, there is a need for stimulating and supporting communication concerning the impact of the change. One way of facilitating such communication is by providing a descriptive process modeling in which business performance is represented "as-is" or "to-be."

This paper proposes a novel supplement to the BPR methodology based on fuzzy cognitive maps (FCMs). This decision aid mechanism proposes a new approach to supplementing the strategic planning and business analysis phases of typical BPR projects, by supporting "intelligent" reasoning of the anticipated ("to-be") business performance. The proposed mechanism utilizes the fuzzy causal characteristics of FCMs as a new modeling technique to generate a hierarchical network of interconnected performance indicators. By using FCMs, the mechanism proposes the development of a causal representation of dynamic business performance principles. Moreover, the proposed mechanism aims at simulating the operational efficiency of complex hierarchical process models with imprecise relationships, while quantifying the impact of the reengineering activities to the overall business architecture. The application of FCMs in modeling the impact of redesign activities to business performance is considered to be novel. In addition, this paper proposes an updated FCM algorithm to model effectively the hierarchical and distributed nature of business models. It is the belief of this paper that the fuzzy reasoning capabilities enhance considerably the usefulness of the proposed mechanism, while reducing the effort for identifying precise performance measurements.

The proposed model has both theoretical and practical benefits. Given the demand for effective redesign of process models, such a succinct mechanism of conveying the essential dynamics of BPR is believed to be useful for anyone contemplating or undertaking a BPR exercise. Primarily, the proposed model targets the principle beneficiaries and stakeholders of performance-driven change (PDC) projects (enterprise administration, redesign leaders, etc.) assisting them to reason effectively about the status of business performance metrics, given the (actual or hypothetical) implementation of a set of process model changes. Nevertheless, the explanatory nature of the mechanism can prove to be useful in a wider educational setting.

This paper consists of six sections. Section II presents a short literature overview of BPR and FCMs. Section III discusses the 
FCM approach in putting realistic and measurable objectives in BPR projects. Section IV presents the actual FCMs and comments on their characteristics. Section V presents the preliminary experimental results of the application of the proposed mechanism. Finally, Section VI concludes this paper and briefly discuses future research activities.

\section{LITERATURE OVERVIEW}

\section{A. BPR Methodologies and Implementations}

1) What is a Business Process: A process can be viewed as a structured series of activities designed to produce a specific business output (product, service, etc.) for a particular customer, internal and/or external demand, etc. This definition implies a strong emphasis on how the actual business is conducted by the enterprise. Processes usually cross the organizational boundaries of an enterprise and occur across or between organizational units.

Processes are generally identified in terms of start - end points, interfaces, organizational units involved, customers served, etc. Processes have organizational entities (e.g., groups, individuals, etc.) as process owners, while performance metrics estimate their effective execution. Typical examples of processes include: development of a new product, procurement of goods from a supplier, creation of a marketing plan, processing and payment of an insurance claim, etc. Typical examples of performance metrics include elapsed time for processing and payment of an insurance claim, production cycle time, number of customer complaints, etc.

2) Limitations of BPR Methodologies: BPR implements a critical analysis and radical redesign of existing business processes so as to achieve breakthrough performance improvements. Most BPR methodologies (e.g., [10], [16], [18], [47], [51], [55]), use diagrammatic notations (like dataflow diagrams, entity relationship attribute techniques, etc.) for modeling business processes. These notations are valuable as informal frameworks in which intuition about the enterprise may be expressed, but they lack the semantic content necessary to drive the process design activities and support reasoning for the effectiveness of the redesigned business model.

Relevant bibliography (e.g., [1], [63], [64]) indicates the majority of business analysis is based on subjective rather than objective analytical methods. There is no formal underpinning to ensure consistency across redesign models. Graphical notations used in business modeling and business redesign do not offer a mechanism for verifying the logical consistency and efficiency of the resulting model. This may create a feeling of insecurity and unnecessary resistance to change. The same bibliography analysis shows that there is a big division in the BPR literature between methodologies that concentrate either on process improvement or on process innovation. The main difference is on the way that change is understood. In the first case, change is performed in an incremental manner whereas in the latter radically. Moreover, most methodologies tend to use a black and white approach. For example, some methodologies regard cost reduction as the major objective, whereas other methodologies regard generic management and the use of information technology (IT) as the main change objective. However, in many cases, a combination of the two approaches has yielded the most impressive results, supporting the argument for the necessity of an integrated holistic view of the enterprise's performance.

Finally, other methodologies use automata theory notations in which a system is modeled by its trajectories through a state space, however, the composition of such models usually leads to the phenomenon known as "state explosion." Petri nets [41] were designed to overcome this problem better than classical automata theory could. Nevertheless, the composition and the combination of business behavior with information structures may still pose severe problems.

3) Why BPR Projects Fail: Major management consulting service providers argue that BPR projects failure is attributed to the following main obstacles: 1) lack of sustained management commitment and leadership; 2) resistance to change; and 3) unrealistic scope and change expectations.

The first two obstacles can be justified by the fact that the top management style and current employees' culture may influence the outcome of process change activities. On the other hand, failure due to unrealistic scope and expectations is attributed to the emphasis given on the tactical (operational) level of BPR projects, compromising the importance and necessity of strategic planning. It is the view of this paper that there are important strategic decisions involved in all major BPR projects. Examples may include the development and prioritization of project objectives, definition of the process model structure, definition of fundamental process assumptions, identification of tradeoffs between process changes, identification of new products and market opportunities, development of human resources strategy, etc.

However, BPR by itself is not a panacea for the enterprise. The challenge for any successful BPR implementation should be to measure an enterprise against best practices and change in a positive manner.

\section{B. Business Process Modeling Attempts}

Once embarked on a BPR initiative, enterprises often employ detailed models to drive redesign activities and communicate the impact of process change to all enterprise levels. The adopted FCM's approach fits within the area of decision support systems [7], [59], [61]. Several modeling approaches can be brought to bear on the task of supplementing various BPR activities. In particular, the field of knowledge-based systems [17], [46] could fulfill the desire for more accurate predictive models.

Among different BPR strategies and methodologies (e.g., [10], [16], [18], [47], [51], [55]), one common feature is to capture existing business models and represent new processes adequately. Research [42] proposed generic structures for modeling business processes. The generic structure possessed two main features suitable for business process modeling: 1) it could represent a business process in various concerns and multiple layers of abstraction and 2) it attempted to lower the barriers between process representation and model analysis by embedding verification and validation with the model. Although these structures do not offer any formal reasoning capabilities, they may serve as a road map for integrating decision support, process modeling and redesign activities. 
Research [6] modeled the impact of process reengineering with system dynamics. It built a model that could describe, explain, and predict gross features of organizational behavior associated with a BPR intervention. Such a model could be used as a decision support tool during the feasibility stage when an organization was considering BPR, or even during the reengineering activities of manufacturing processes [20]. Research [6] built on an earlier research attempt to use system dynamics for modeling the interaction between competitive capabilities of quality and cost during total quality management (TQM) initiatives [5]. While the model interpreted competitive capabilities as a network of causal relationships, it only utilized a small set of performance factors having the "level of specialization" as the only key driver. This model did not cascade relationships into smaller groupings of competitive capabilities, nor did it allow the connection of the submodels. Finally, the model required formal definition of causal relationships (e.g., functions), which posed a significant overhead in supplementing the BPR exercise.

With risk defined as the possibility of deviation in the results from the expected goals, BPR initiatives clearly involve risk taking. Research [9] reported the development of a tool to quantitatively estimate the potential risk level of a BPR effort before an organization commits its resources to that effort. The underlying research employed a survey of BPR-experienced organizations to collect assessment information in order to build a BPR risk estimation model. The tool used triangular fuzzy numbers to approximate the degree of success/failure of proposed BPR initiatives. While the tool based its reasoning capabilities on simple arithmetic approximations, it could be applied by enterprises contemplating BPR, thus giving such organizations a heretofore unavailable estimate of the risk level of proposed BPR efforts. The utilization of uncertainty was also suggested by Jones and Ryan [22], which proposed a contingency model of quality management practices, whereby quality management orientation, process choice, and environmental uncertainty were the contextualizing variables.

Other research attempts built on theories like nonlinearity to reason about the impact of process change to the business model of the enterprise. Research [48] reported such experience in applying nonlinear systems theory in health care settings. This research revealed that nonlinear science offered a practical new frame of reference for BPR initiatives. It discussed practical insights offered by nonlinear systems theory and provided a clinical example of multidimensional thinking as applied in an acute care setting.

Research [35] proposed a cognitive map-based method to support business process performance measurement. It offered a two-phase cognitive modeling (called TCM), to help organizational members identify potential organizational conflicts and capture core business activities rather than drive process redesign by reasoning on the impact of redesign activities. To apply the method in the real-world context, a prototype modeling tool was developed. This mechanism generated local and global maps to model partial functional interrelations within an organization. Research [35] proposed a number of informal/ambiguous techniques to generate and validate the organizational cognitive maps, like interviews, observation, group discussions, questionnaires, document analysis, and so forth. Causal values were generated according to the pair wise comparison technique, but no fuzzy definitions were allowed. To this extend, A-Pool and MIND [35] are the only two articulated frameworks that allowed direct weight assignment.

To make the issue more understandable it is the belief of this paper that there is no other tool that allows the user to integrate FCM simulation into BPR exercises with the functionality and characteristics of the proposed mechanism (presentation will follow in Sections III and IV). TCM, for example, may supplement BPR by drawing FCMs with allowed node values of 0 and 1 and no dynamic simulation capabilities. Frameworks like MIND, SODA, TCM, and COCOMAP (all compared in [35]) provided methodologies and guidance that allowed the user to perform FCM analysis by identifying node conflicts in multiple maps, loops, cycles, etc. However, nodes in different maps could not be linked dynamically to create map hierarchies.

Accurate predictive models may already exist in BPR consultancies. Through their experiences they are likely to have built up databases that could underpin more detailed approaches such as case-based reasoning. Unfortunately, the existence and internal features of these models are more likely to remain confidential, given the commercial sensitivity of such knowledge.

\section{Fuzzy Cognitive Maps (FCMs)}

1) Definitions and Algorithms: FCMs is a modeling methodology for complex decision systems, which originated from the combination of fuzzy logic [67] and neural networks. An FCM describes the behavior of a system in terms of concepts; each concept represents an entity, a state, a variable, or a characteristic of the system [11].

Kosko in [31] defined a concept $\boldsymbol{C}_{\boldsymbol{i}}$ that constitutes causal relationships in FCM as follows:

$$
C_{i}=\left(Q_{i} \cup \sim Q_{i}\right) \cap M_{i}
$$

where $\boldsymbol{Q}_{i}$ is a quantity fuzzy set and $\sim \boldsymbol{Q}_{i}$ is a disquantity fuzzy set. $\sim \boldsymbol{Q}_{i}$ is the negation of $\boldsymbol{Q}_{i}$. Each $\boldsymbol{Q}_{i}$ and $\sim \boldsymbol{Q}_{i}$ partitions the whole set $\boldsymbol{C}_{i}$. Double negation $\sim \boldsymbol{Q}_{i}$ equals to $\boldsymbol{Q}_{i}$, implying that $\sim \boldsymbol{Q}_{i}$ corresponds to $\boldsymbol{Q}_{i}^{c}$, the complement of $\boldsymbol{Q}_{i}$. However, negation does not mean antonym. Therefore, if a disquantity fuzzy set $\sim \boldsymbol{Q}_{i}$ does not correspond to the complement of $\boldsymbol{Q}_{i}$, we will call it as anti-quantity fuzzy set to clarify the subtle meaning in the disquantity fuzzy set, as proposed by [27]. $\boldsymbol{M}_{\boldsymbol{i}}$ is a modifier fuzzy set that modifies $\boldsymbol{Q}_{i}$ or $\sim \boldsymbol{Q}_{i}$ concretely. The modifier fuzzy set fuzzily intersects the fuzzy union of a quantity fuzzy set and a disquantity fuzzy set.

Kosko in [31] also formally defined the positive and negative fuzzy causal relationships (or fuzzy causality) as follows.

- Definition 1. $\boldsymbol{C}_{\boldsymbol{i}}$ causes $\boldsymbol{C}_{j}$ iff $\left(\boldsymbol{Q}_{i} \cap \boldsymbol{M}_{i}\right) \subset\left(\boldsymbol{Q}_{j} \cap \boldsymbol{M}_{j}\right)$ and $\left(\sim \boldsymbol{Q}_{i} \cap \boldsymbol{M}_{i}\right) \subset\left(\sim \boldsymbol{Q}_{j} \cap \boldsymbol{M}_{j}\right)$

- Definition 2. $\boldsymbol{C}_{i}$ causally decreases $\boldsymbol{C}_{j}$ iff $\left(\boldsymbol{Q}_{i} \cap \boldsymbol{M}_{i}\right) \subset$ $\left(\sim \boldsymbol{Q}_{j} \cap \boldsymbol{M}_{j}\right)$ and $\left(\sim \boldsymbol{Q}_{i} \cap \boldsymbol{M}_{i}\right) \subset\left(\boldsymbol{Q}_{j} \cap \boldsymbol{M}_{j}\right)$

Here, " $\subset$ " stands for fuzzy set inclusion (logical implication).

A more insightful and practical definition of FCMs follows. FCM nodes are named by concepts forming the set of concepts $\boldsymbol{C}=\left\{\boldsymbol{C}_{1}, \boldsymbol{C}_{2}, \ldots, \boldsymbol{C}_{n}\right\}$. $\operatorname{Arcs}\left(\boldsymbol{C}_{j}, \boldsymbol{C}_{\boldsymbol{i}}\right)$ are oriented and represent causal links between concepts; that is how concept $C_{j}$ causes concept $C_{i}$. Arcs are elements of the set 


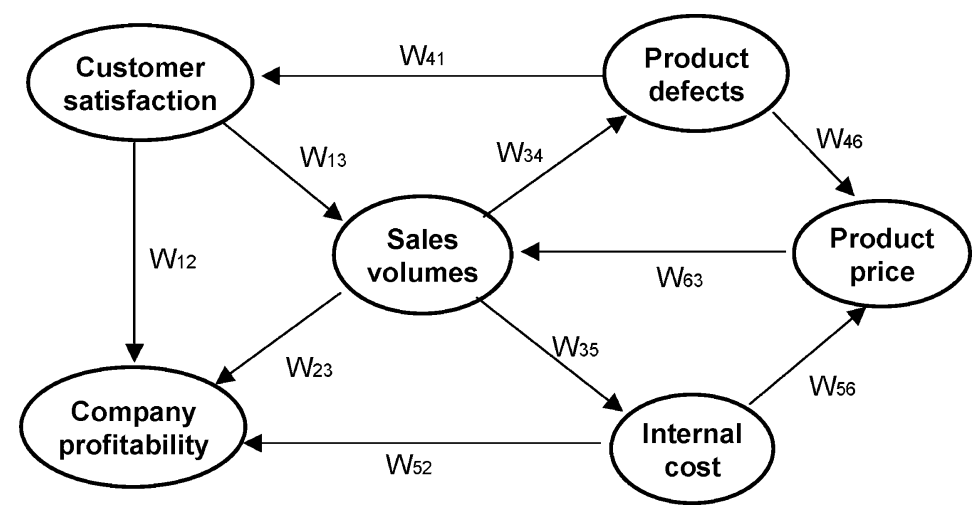

Fig. 1. Simple FCM.

$\boldsymbol{A}=\left\{\left(\boldsymbol{C}_{j}, \boldsymbol{C}_{i}\right)_{j i}\right\} \subset \boldsymbol{C} \times \boldsymbol{C}$. Weights of arcs are associated with a weight value matrix $\boldsymbol{W}_{n x n}$, where each element of the matrix $\boldsymbol{w}_{j i} \in[-\mathbf{1}, \ldots, \mathbf{1}] \subset \boldsymbol{R}$ such that if $\left(\boldsymbol{C}_{j}, \mathbf{C}_{i}\right) \notin A$, then $\boldsymbol{w}_{j i}=0$ else excitation (respectively, inhibition) causal link from concept $\boldsymbol{C}_{j}$ to concept $\boldsymbol{C}_{\boldsymbol{i}}$ gives $\boldsymbol{w}_{j i}>0$ (respectively, $\left.\boldsymbol{w}_{j i}<0\right)$. The proposed methodology framework assumes that $[-1, \ldots, 1]$ is a fuzzy bipolar interval, bipolarity being used as a means of representing a positive or negative relationship between two concepts.

In practice, the graphical illustration of an FCM is a signed graph with feedback, consisting of nodes and weighted interconnections (e.g., $\stackrel{\text { Weight }}{\longrightarrow}$ ). Signed and weighted arcs (elements of the set $A$ ) connect various nodes (elements of the set $C$ ) representing the causal relationships that exist among concepts. This graphical representation (e.g., Fig. 1) illustrates different aspects in the behavior of the system, showing its dynamics [31] and allowing systematic causal propagation (e.g., forward and backward chaining). Positive or negative sign and fuzzy weights model the expert knowledge of the causal relationships [32]. Concept $\boldsymbol{C}_{j}$ causally increases $\boldsymbol{C}_{i}$ if the weight value $\boldsymbol{w}_{j i}>0$ and causally decreases $\boldsymbol{C}_{i}$ if $\boldsymbol{w}_{j i}<0$. When $\boldsymbol{w}_{j i}=0$, concept $\boldsymbol{C}_{j}$ has no causal effect on $\boldsymbol{C}_{i}$. The sign of $\boldsymbol{w}_{j i}$ indicates whether the relationship between concepts is positive $\left(C_{j} \stackrel{W_{j, i}}{\longrightarrow} C_{i}\right)$ or negative $\left(\boldsymbol{C}_{j} \stackrel{\boldsymbol{W}_{j, i}}{\longrightarrow} \sim \boldsymbol{C}_{i}\right)$, while the value of $\boldsymbol{w}_{j i}$ indicates how strongly concept $\boldsymbol{C}_{j}$ influences concept $\boldsymbol{C}_{i}$. The forward or backward direction of causality indicates whether concept $\boldsymbol{C}_{j}$ causes concept $\boldsymbol{C}_{i}$ or vice versa.

Simple variations of FCMs mostly used in business decision-making applications may take trivalent weight values $[-1,0,1]$. This paper allows FMCs to utilize fuzzy word weights like strong, medium, or weak, each of these words being a fuzzy set to provide complicated FCMs. In contrast, Kwahk and Kim [35] adopted only a simple relative weight representation in the interval $[-1, \ldots, 1]$. To this extend, Kwahk and Kim [35] offered reduced functionality since it does not allow fuzzy weight definitions.

Generally speaking, FCM concept activations take their value in an activation value set $V=\{0,1\}$ or $\{-1,0,1\}$ if in crisp mode or $[-\delta, \mathbf{1}]$ with $\boldsymbol{\delta}=\mathbf{0}$ or $\mathbf{1}$ if in fuzzy mode. The proposed methodology framework assumes fuzzy mode with $\delta=1$, however, crisp activation sets are also supported. At step t $\in N$, each concept $\boldsymbol{C}_{j}$ is associated with an inner activation value $\boldsymbol{a}_{j}^{t} \in \boldsymbol{V}$, and an external activation value $\boldsymbol{e}_{a_{j}}^{t} \in \boldsymbol{R}$.
FCM is a dynamic system. Initialization is $\boldsymbol{a}_{j}^{0}=0$. The dynamic obeys a general recurrent relation $\boldsymbol{a}^{t+1}=$ $\boldsymbol{f}\left(\boldsymbol{g}\left(\boldsymbol{e}_{a}^{t}, \boldsymbol{W}^{T} \boldsymbol{a}^{t}\right)\right), \forall \mathrm{t} \geq 0$, involving weight matrix product with inner activation, fuzzy logical operators $(\boldsymbol{g})$ between this result and external forced activation and finally normalization $(f)$. However, this paper assumes no external activation (hence, no fuzzy logical operators), resulting to the following typical formula for calculating the values of concepts of FCM

$$
\boldsymbol{a}_{i}^{t+1}=\boldsymbol{f}\left(\sum_{j=1, j \neq i}^{n} w_{j i} \boldsymbol{a}_{j}^{t}\right)
$$

where $\boldsymbol{a}_{i}^{t+1}$ is the value of concept $\boldsymbol{C}_{i}$ at step $\boldsymbol{t}+1, \boldsymbol{a}_{j}^{t}$ the value of the interconnected concept $\boldsymbol{C}_{j}$ at step $\boldsymbol{t}, \boldsymbol{w}_{j i}$ is the weighted arc from $\boldsymbol{C}_{j}$ to $\boldsymbol{C}_{i}$ and $f: \boldsymbol{R} \rightarrow \boldsymbol{V}$ is a threshold function, which normalizes activations. Two threshold functions are usually used. The unipolar sigmoid function where $\lambda>0$ determines the steepness of the continuous function $\boldsymbol{f}(\boldsymbol{x})=$ $1 /\left(1+e^{-\lambda x}\right)$. When concepts can be negative $(\delta<0)$, function $\boldsymbol{f}(\boldsymbol{x})=\tanh (\boldsymbol{x})$ is used.

To understand better the analogy between the sign of the weight and the positive/negative relationship, it may be necessary to revisit the characteristics of fuzzy relation [26], [39]. A fuzzy relation from a set $\boldsymbol{A}$ to a set $B$ or $(\boldsymbol{A}, \boldsymbol{B})$ represents its degree of membership in the unit interval $[\mathbf{0 , 1}]$. Generally speaking, sets $\boldsymbol{A}$ and $\boldsymbol{B}$ can be fuzzy sets. The corresponding fuzzy membership function is $\boldsymbol{\mu} f: \boldsymbol{A} \times \boldsymbol{B} \rightarrow[0,1]$. Therefore, $\boldsymbol{\mu f}(\boldsymbol{x}, \boldsymbol{y})$ is interpreted as the "strength" of the fuzzy membership of the fuzzy relation $(\boldsymbol{x}, \boldsymbol{y}$, where $\boldsymbol{x} \in \boldsymbol{A}$ and $\boldsymbol{y} \in \boldsymbol{B}$. Then, this fuzzy relation concept can be denoted equivalently as $x \stackrel{\mu f}{\longrightarrow} y$ and applied to interpret the causality value of FCM, since $\boldsymbol{w}_{j i}$ (the causality value of the arc from nodes $\boldsymbol{C}_{j}$ to $\boldsymbol{C}_{i}$ ) in a certain FCM is interpreted as the degree of fuzzy relationship between two nodes $\boldsymbol{C}_{j}$ and $\boldsymbol{C}_{\boldsymbol{i}}$. Hence, $\boldsymbol{w}_{j i}$ in FCMs is the fuzzy membership value $\boldsymbol{\mu} f\left(\boldsymbol{C}_{j}, \boldsymbol{C}_{i}\right)$ and can be denoted as $\boldsymbol{C}_{j} \stackrel{\boldsymbol{w}_{j, i}}{\longrightarrow} \boldsymbol{C}_{i}$.

However, we understand that the fuzzy relation (weight) between concept nodes is more general than the original fuzzy relation concept. This is because it can include negative (-) fuzzy relations. Fuzzy relations mean fuzzy causality; causality can have a negative sign. In FCMs, the negative fuzzy relation (or causality) between two concept nodes is the degree of a relation with a "negation" of a concept node. For example, 
if the negation of a concept node $C_{i}$ is noted as $\sim C_{i}$, then $\boldsymbol{\mu} \boldsymbol{f}\left(\boldsymbol{C}_{\mathrm{j}}, \boldsymbol{C}_{\mathrm{i}}\right)=-0.6$ means that $\boldsymbol{\mu} \boldsymbol{f}\left(\boldsymbol{C}_{\mathrm{j}}, \sim \boldsymbol{C}_{i}\right)=0.6$. Conversely, $\boldsymbol{\mu} f\left(\boldsymbol{C}_{\mathbf{j}}, \boldsymbol{C}_{\mathbf{i}}\right)=0.6$ means that $\boldsymbol{\mu} \boldsymbol{f}\left(\boldsymbol{C}_{\mathbf{j}}, \sim \boldsymbol{C}_{i}\right)=-0.6$.

FCMs help to predict the evolution of the system (simulation of behavior) and can be equipped with capacities of hebbian learning [30], [33]. FCMs are used to represent and to model the knowledge on the examining system. Existing knowledge of the behavior of the system is stored in the structure of nodes and interconnections of the map. The fundamental difference between FCMs and a neural networks is in the fact that all the nodes of the FCM graph have a strong semantic defined by the modeling of the concept, whereas the nor input/nor output nodes of the graph of the neural network have a weak semantic, only defined by mathematical relations.

2) Applications of FCMs: Over the last ten years, a variety of FCMs have been used for representing knowledge and artificial intelligence in engineering applications, like geographical information systems [44] and fault detection [49], [53]. FCMs have been used in modeling the supervision of distributed systems [62]. FCMs have also been used in operation research [8], web data mining [19], [39], as a back end to computer-based models and medical diagnosis (e.g., [14]).

Several research reports applying basic concepts of FCMs have also been presented in the field of business and other social sciences. References [2] and [54] have used FCM for representing tacit knowledge in political and social analysis. FCMs have been successfully applied to various fields such as decision making in complex war games [29], strategic planning [12], [57], strategic information systems planning [25], information retrieval [21], and distributed decision modeling [68]. Research [37] has successfully applied FCMs to infer implications from stock market analysis results. Research [38] has also suggested a new concept of fuzzy causal relations found in FCMs and has applied it to analyze and predict stock market trends. The inference power of FCMs has also been adopted to analyze the competition between two companies, which have been assumed to use differential games mechanisms to set up their own strategic planning [40]. FCMs have been integrated with case-based reasoning technique to build organizational memory in the field of knowledge management [50]. Research [52] proposed the use of FCM a tool to model emotional behavior of virtual actors improvising in free interaction within the framework of a "nouvelle vague" scenario, and discussed the problem of delocalizing each agent level to model autonomous agents within a virtual world. Recent research adopted FCMs to support the core activities of highly technical functions like urban design [66]. Summarizing, FCMs can contribute to the construction of more intelligent systems, since the more intelligent a system becomes, the more symbolic and fuzzy its representations become.

In addition, a few modifications have been proposed. For example, Silva [60] has proposed new forms of combined matrices for FCMs, Hagiwara [15] has extended FCMs by permitting nonlinear and time delay on the arcs, research [58] has presented a method for automatically constructing FCMs. More recently, Liu and Satur [44] has carried out extensive research on FCMs, investigating inference properties of FCMs and has proposed contextual FCMs based on the object-oriented paradigm of decision support, having applied contextual FCMs to geographical information systems [43].

\section{Putting Realistic And Measurable ObJectives TO BPR}

\section{A. Selection of Performance Indicators}

Setting redesign objectives without some form of measurement, targeting and monitoring may not lead to the expected performance improvement [34]. If the enterprise is not able to reason about the degree and impact of process change, then the enterprise may not be able to achieve the expected performance goals. It is the view of this paper that in order to quantify effectively the impact of the reengineering activities to the business model, the key performance indicators (KPIs) should comply with the following selection rules.

- KPIs should be limited to what is essential and suitable for the target market group. Enterprises often think that all gaps between current performance and customer requirements require action; they think that every effort should be made to meet all customer needs. It is the view of this paper that the enterprise must decide on its customer focus first and then allocate its resources accordingly.

- KPIs should monitor core business first. Core processes are defined to be how the enterprise transforms its inputs into outputs to satisfy customer needs. Core business offers a good match between the enterprise's strengths and customer requirements, reasonable resource allocation demands, as well as a competitive market position.

- KPIs should allow regular monitoring, furthermore, KPIs should be agreed with major enterprise stakeholders who can influence performance change positively.

The selection of such KPIs does necessarily limit the change capabilities of the enterprise. On the contrary, it is the belief of this paper that this selection provides an efficient framework for assessing the impact of redesign decisions. As an example, there may be occasions of a highly attractive potential market but resource requirements for achieving competitiveness in this market may be also equally significant. Will the enterprise risk moving into nonprofitable business activities, or will the enterprise excel in core business activities, consolidating its position through improved performance? The utilization of such performance indicators allows the enterprise to comprehend fully such interrelated implications of business changes, and follow a step-by-step approach before deciding upon radical redesign initiatives.

\section{B. FCM as a Supplement During BPR Projects}

A typical BPR methodology consists of a series of phases for redesigning the process model of an enterprise:

- Phase 1: strategic BPR planning;

- Phase 2: business modeling;

- Phase 3: business analysis;

- Phase 4: redesign;

- Phase 5: continuous improvement.

The mechanism proposed by this paper focuses on supplementing a typical BPR methodology in Phases 1 and 3. During the implementation of these phases, a typical BPR methodology defines the strategic BPR planning performance metrics (strategic level metrics) and the business analysis performance 
BPR

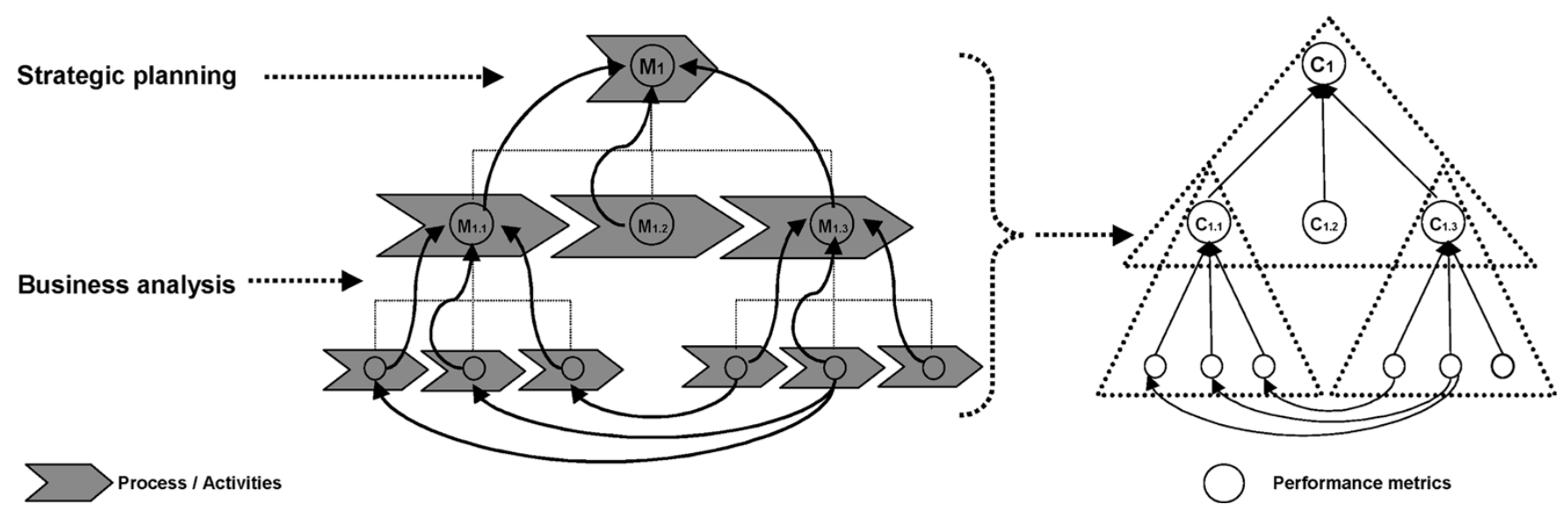

Fig. 2. Supplementing BPR projects with FCMs.

metrics (operational-tactical level metrics) that measure the performance of the redesigned enterprise. Such metrics present inherent relationships with the process or organizational hierarchy of the enterprise.

In practice, strategic business metrics must cascade to operational metrics to allow the redesigner to comprehend inherent relations among the different processes and organizational levels of the enterprise. Similarly, performance metrics of partial activities of a process must propagate up the overall performance metrics of the process itself. However, relationships between metrics at the same level or even relationships between metrics of different levels with no apparent relationships are not always clear and well defined. Thus, reasoning of the chained impact of performance metrics to the performance of the overall business model is not always feasible.

This paper proposes the utilization of such metrics (Fig. 2) to develop the FCMs and reason about the performance of existing ("as-is") and desired ("to-be") business models. The information yielded by this reasoning mechanism can be seen as a decision aid to direct the redesign phase (fourth phase) of the BPR methodology toward efficient process change.

The proposed mechanism utilizes FCMs to interpret the following:

- business performance metrics as concepts (graphically represented as nodes);

- decision weights as relationship weights (graphically represented as arrowhead lines);

- decision variables as concept values;

- hierarchical decomposition (top-down decomposition) of strategic BPR planning metrics to operational metrics and constituent submetrics as a hierarchy of FCMs; this interpretation allows the redesigners to reason about lower level FCMs first (constituent metrics) before they reason about higher level business metric (affected metrics).

The proposed mechanism supports the reasoning about the overall or partial business model performance using performance indicators from the BPR philosophy. In contrast to [35], the proposed mechanism builds on hierarchical performance metrics interrelationships identified and utilized by the
BPR methodology. The proposed approach does not perform or guide the redesigners to perform any stage of the BPR methodology. Also, the approach does not perform or guide the redesigners to estimate the absolute value of any of the performance metrics and/or the overall business performance. It only allows the stakeholders (enterprise management, redesigners, etc.) to reason about the qualitative state of business metrics using fuzzy linguistic variables like high-neutral-low cost, high-neutral-low impact of IT infrastructure to cost, etc.

\section{Development of FCMs}

1) FCM Development Roadmap: The creation of FCMs and their incorporation into BPR projects is materialized through a series of interpretation steps. Each of the proposed steps requires input to be provided, interpretation tasks to be performed, and outcome to be produced. Fig. 3 presents the generic FCM development activities in the form of a simplified flowchart. This chart assumes that a typical BPR methodology has already generated initial strategic BPR plans and business models.

2) New FCM Algorithm: This paper extends the basic FCM algorithm (as discussed in Section II-C1 and also used by [35]), by proposing the following new FCM algorithm:

$$
\boldsymbol{a}_{i}^{t+1}=f\left(\boldsymbol{k}_{1} a_{i}^{t}+\boldsymbol{k}_{2} * \sum_{j=1, j \neq i}^{n} \boldsymbol{w}_{j i} \boldsymbol{a}_{j}^{t}\right) .
$$

This paper assumes that coefficients $\boldsymbol{k}_{1}$ and $\boldsymbol{k}_{2}$ can be fuzzy sets.

Coefficient $\boldsymbol{k}_{1}$ represents the proportion of the contribution of the value of the concept $\boldsymbol{a}_{i}$ at time $\boldsymbol{t}$ in the computation of the value of $\boldsymbol{a}_{i}$ at time $\boldsymbol{t}+1$. In practice, this is equivalent to assume that $\boldsymbol{w}_{i i}=\boldsymbol{k}_{1}$. The incorporation of this coefficient results in smoother variation of concept values during the iterations of the FCM algorithm.

Coefficient $\boldsymbol{k}_{2}$ expresses the "influence" of the interconnected concepts in the configuration of the value of the concept $a_{i}$ at time $t+1$. It is the proposal of this paper that such a coefficient 


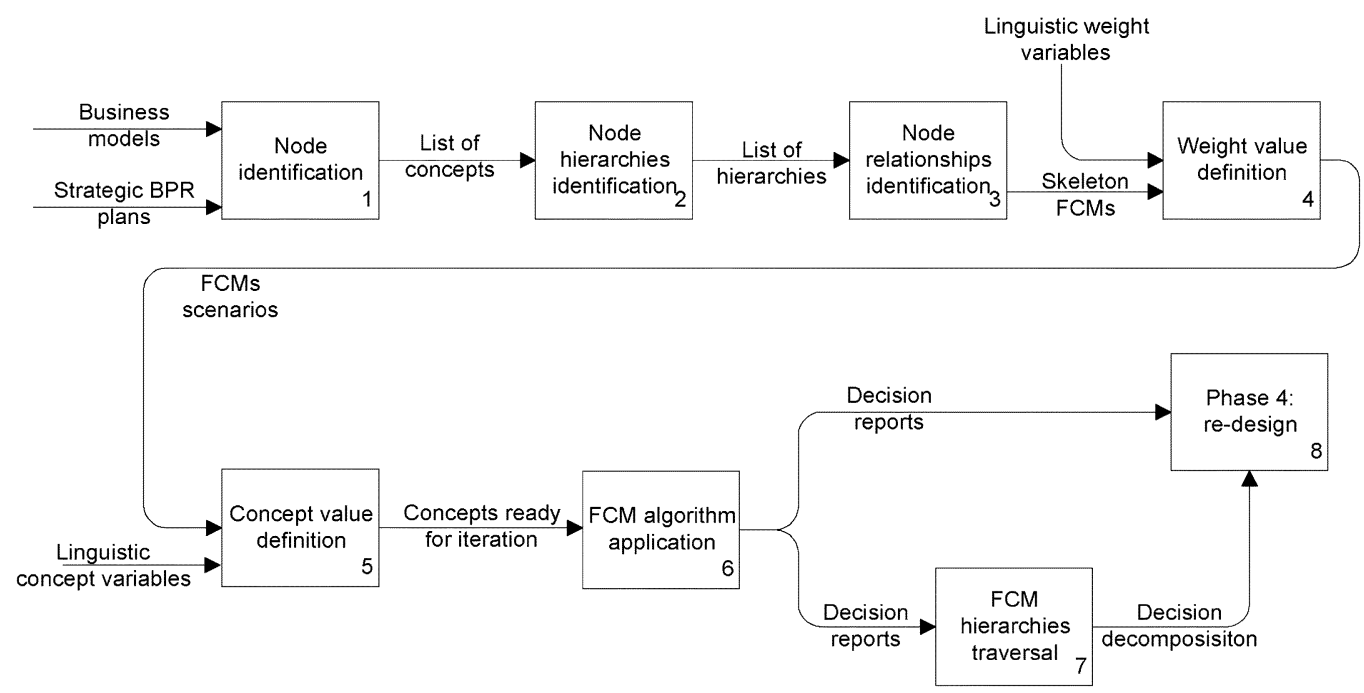

Fig. 3. FCM development tasks.

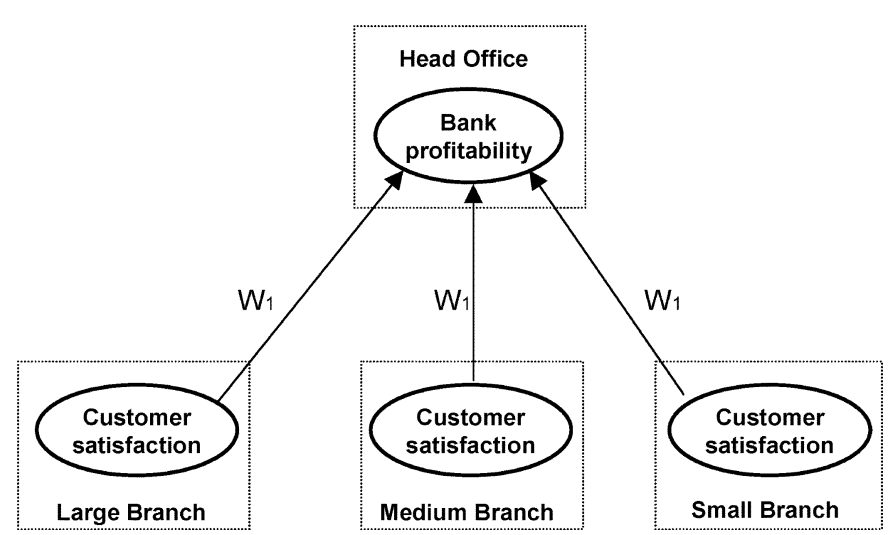

Fig. 4. Duplicate relationships between distributed organizational units.

should be used to align indirectly causal relationships (essentially, the value of concept $\boldsymbol{C}_{i}$ ) with the centralized/decentralized nature of concept $\boldsymbol{C}_{j}$, as well as with the significance of the hierarchical positioning of concept $C_{j}$ within the enterprise.

Intuitively, the introduction coefficient $\boldsymbol{k}_{2}$ imposes three steps of analysis for establishing the "influence" of causal relationships.

Step 1) Estimation of the direct influence of a concept $\boldsymbol{C}_{j}$ to another concept $\boldsymbol{C}_{i}$ with the weight $\left(\boldsymbol{w}_{j i}\right)$ of the relationship. Both $\boldsymbol{C}_{i}$ and $\boldsymbol{C}_{j}$ should belong to the same organizational (or process) level, that is $\boldsymbol{k}_{2}=1$.

Step 2) Approximation of the indirect importance of duplicate causal relationships spanning horizontally to duplicate organizational (or process) units using coefficient $\boldsymbol{k}_{2}<1$. Similarly, for distributed organizational (or process) units.

Step 3) Approximation of the indirect importance of causal relationships spanning vertically to different organizational (or process) levels also using coefficient $\boldsymbol{k}_{2}<1$.

Consider, for example, a typical bank with a typical branch network operating with the same process model (Fig. 4). Let

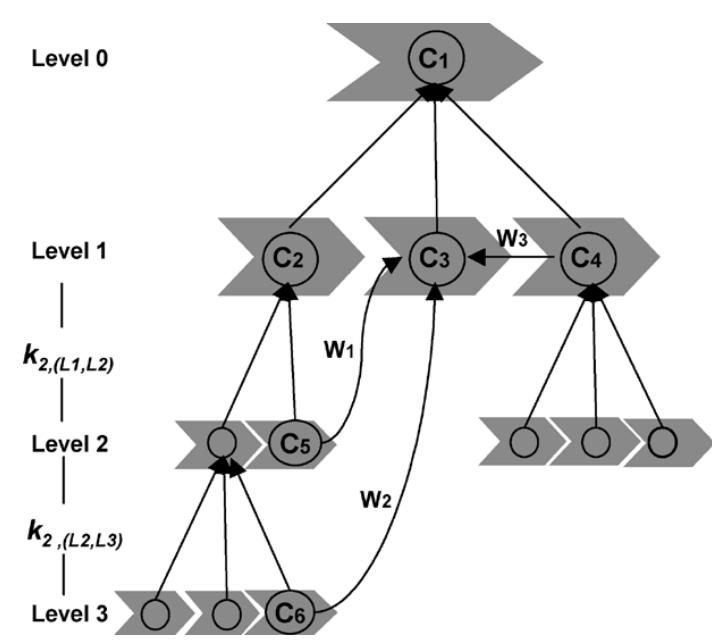

Fig. 5. Relationships spanning vertically to different process levels.

"customer satisfaction" and "bank productivity" be interrelated performance concepts. From a theoretical standpoint weight $\mathrm{w}_{1}$ should appear to be the same for all duplicate "customer satisfaction-bank profitability" relationships across all branches. From a practical standpoint, branches may serve different number of customers (or even customers with different transaction volumes). In this example, coefficient $\boldsymbol{k}_{2}$ models the fact that customer satisfaction in a large branch which serves many customers (or even few customers with large transaction volumes) is more important to the profitability of the bank in comparison to the customer satisfaction in a small branch which serves few customers (or even customers with very small transaction volumes), even if customer satisfaction is the same for all branches.

Similarly, Fig. 5 presents a generic process breakdown structure, accompanied with a sample concept hierarchy. Regardless of weight values $w_{1}, w_{2}, w_{3}$, coefficients $1=\boldsymbol{k}_{2,(L 1, L 1)} \geq \boldsymbol{k}_{2,(L 1, L 2)} \geq \boldsymbol{k}_{2,(L 2, L 3)}$ model the fact that affecting concepts at level $L_{i}$ (e.g., concept $C_{5}$ ) are more important in determining the value of affected concepts at level $L_{i+1}$ (e.g., concept $C_{3}$ ) in comparison to other affecting 


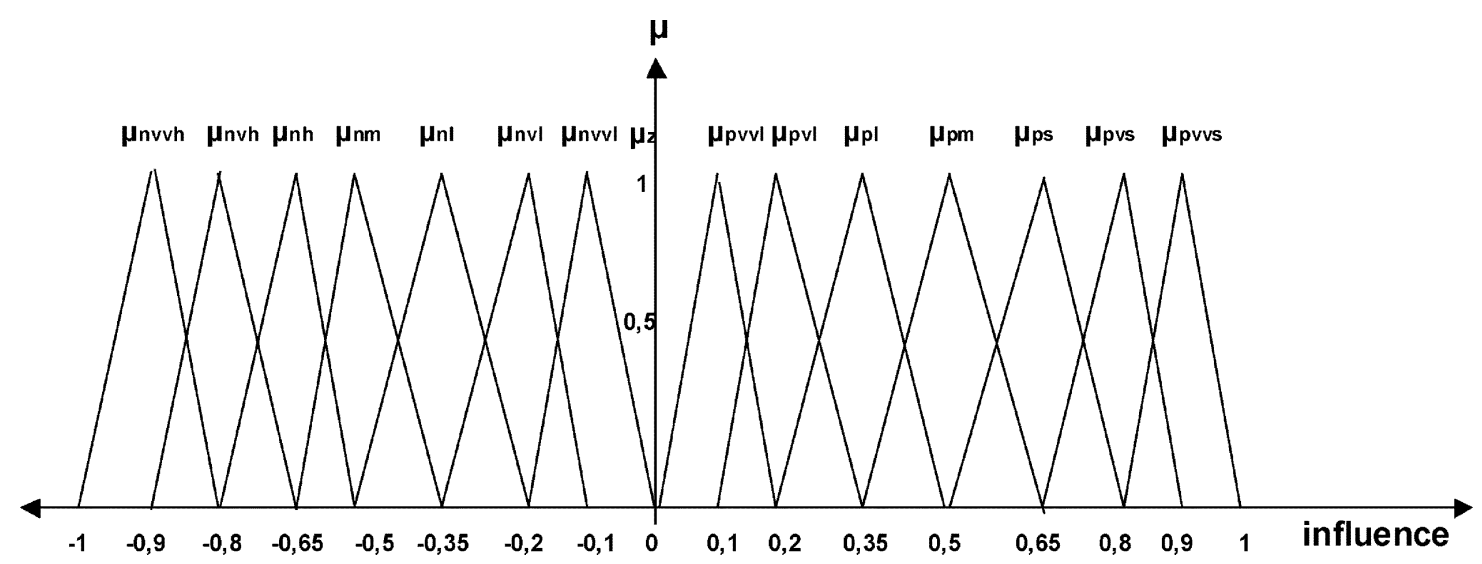

Fig. 6. Membership functions of linguistic variable influence.

concepts at level $L_{i-1}$ (e.g., concept $C_{6}$ ). $\boldsymbol{k}_{2,(L i, L j)}$ stands for the value of coefficient $k_{2}$ associated with levels $i$ and $j$.

Ideally, coefficient $k_{2}$ could break down into two separate coefficients (say $\boldsymbol{k}_{2}=\boldsymbol{x} * \boldsymbol{k}_{2}^{x}+\boldsymbol{y} * \boldsymbol{k}_{2}^{y}$ ), where $\boldsymbol{k}_{2}^{x}$ aligns indirectly the value of concept $\boldsymbol{C}_{i}$ with the centralized/decentralized nature of concept $\boldsymbol{C}_{j}$, while $\boldsymbol{k}_{2}^{y}$ aligns indirectly the value of concept $\boldsymbol{C}_{i}$ with the significance of the hierarchical positioning of concept $\boldsymbol{C}_{j}$ within the enterprise. Parameters $\boldsymbol{x}, \boldsymbol{y}$ could present the relative importance of $\boldsymbol{k}_{2}^{x}$ and $\boldsymbol{k}_{2}^{y}$ in mixed interconnection problems (e.g., concepts in different process levels participating in duplicate relationships). However, preliminary experiments showed that this separation imposed unnecessary initialization overheads (see Section III-D) without increasing significantly the accuracy of the FCM algorithm.

Two alternative but equally interesting interpretations of coefficient $k_{2}$ follow.

- If the set of identified performance concepts $\boldsymbol{C}_{j}, j \neq i$, is incomplete (e.g., incomplete maps, missing concepts, etc.), then the estimation of the value of concept $\boldsymbol{C}_{i}$ may prove imprecise. In this case, coefficient $\boldsymbol{k}_{2}$ may indicate the sufficiency of the set of concepts $C_{j} j \neq i$, in the calculation of the value of the concept $\boldsymbol{C}_{i}$.

- If the information necessary to approximate the input values of concepts $\boldsymbol{C}_{j}, j \neq i$, is incomplete (e.g., incomplete customer satisfaction survey), then the estimation of the value of concept $C_{i}$ may also prove imprecise. In this case, coefficient $\boldsymbol{k}_{2}$ may indicate the completeness of information utilized in the approximation of the input values of concepts $C_{j}$ during the calculation of the value of the concept $\boldsymbol{C}_{i}$.

\section{Assigning Variables to FCM Weights and Concepts}

1) Expert Linguistic Variables: The third activity ("node relationships identification") generates skeleton FCMs. Based on these skeleton FCMs, the redesigner can generate several business cases (scenarios), each modifying the fuzzy weight value of the association rules, as well as coefficients $\boldsymbol{k}_{1}$ and $\boldsymbol{k}_{2}$. The third activity receives as input linguistic variables to define the fuzzy weights in the fuzzy bipolar interval $[-1, \ldots, 1]$. Then, the redesigner can reason about the business performance (activity 5) by inputting linguistic variables to define the concept values in the fuzzy bipolar interval $[-1, \ldots, 1]$ and trigger the FCM algorithm. This approach offers independent reasoning of each weight value, rather than estimation of the relative (i.e., dependent) "strength" as suggested by [35].

In order to define weight value of the association rules the following methodology is proposed. Experts are asked to describe the interconnection influence of concepts using linguistic notions. Influence of one concept over another, is interpreted as a linguistic variable in the interval $[-1,1]$. Its term set $\mathbf{T}$ (influence) is

\section{$\mathbf{T}($ influence $)$}

$=\{$ negatively very-very high, negatively very high, negatively high, negatively medium, negatively low, negatively very low, negatively very-very low, zero, positively very-very low, positively very low, positively low, positively medium, positively high, positively very high, positively very-very high .

This paper proposes a semantic rule $\boldsymbol{M}$ to be defined at this point. The above-mentioned terms are characterized by the fuzzy sets whose membership functions $\mu$ are shown in Fig. 6.

- $M$ (negatively very-very high)

$=$ the fuzzy set for "an influence close to $-90 \%$ " with membership function $\boldsymbol{\mu}_{\boldsymbol{n} \boldsymbol{v v \boldsymbol { h }}}$.

- $M$ (negatively very high)

$=$ the fuzzy set for "an influence close to $-80 \%$ " with membership function $\boldsymbol{\mu}_{\boldsymbol{n v \boldsymbol { h }}}$.

- $M$ (negatively high)

$=$ the fuzzy set for "an influence close to $-65 \%$ " with membership function $\boldsymbol{\mu}_{\boldsymbol{n} \boldsymbol{h}}$.

- $M$ (negatively medium)

$=$ the fuzzy set for "an influence close to $-50 \%$ " with membership function $\boldsymbol{\mu}_{n \boldsymbol{m}}$.

- $M$ (negatively low)

$=$ the fuzzy set for "an influence close to $-35 \%$ " with membership function $\boldsymbol{\mu}_{n l}$.

- $M$ (negatively very low)

$=$ the fuzzy set for "an influence close to $-20 \%$ " with membership function $\boldsymbol{\mu}_{\boldsymbol{n v l} \boldsymbol{l}}$. 
- $M$ (negatively very-very low)

$=$ the fuzzy set for "an influence close to $-10 \%$ " with membership function $\boldsymbol{\mu}_{\boldsymbol{u v v l}}$.

- $M($ zero $)$

$=$ the fuzzy set for "an influence close to 0" with membership function $\boldsymbol{\mu}_{\boldsymbol{z}}$.

- $M$ (positively very-very low)

$=$ the fuzzy set for "an influence close to $10 \%$ " with membership function $\boldsymbol{\mu}_{\boldsymbol{p} \boldsymbol{v} \boldsymbol{v}}$.

- $M$ (positively very low)

$=$ the fuzzy set for "an influence close to 20\%" with membership function $\boldsymbol{\mu}_{p v \boldsymbol{l}}$.

- $M$ (positively low)

$=$ the fuzzy set for "an influence close to 35\%" with membership function $\boldsymbol{\mu}_{\boldsymbol{p l}}$.

- $M$ (positively medium)

$=$ the fuzzy set for "an influence close to 50\%" with membership function $\boldsymbol{\mu}_{p m}$.

- $M$ (positively high)

$=$ the fuzzy set for "an influence close to 65\%" with membership function $\boldsymbol{\mu}_{\boldsymbol{p h}}$.

- $M$ (positively very high)

$=$ the fuzzy set for "an influence close to 80\%" with membership function $\boldsymbol{\mu}_{\boldsymbol{p} \boldsymbol{v} \boldsymbol{h}}$.

- $M$ (positively very-very high)

$=$ the fuzzy set for "an influence close to 90\%" with membership function $\boldsymbol{\mu}_{\boldsymbol{p} v \boldsymbol{v} \boldsymbol{h}}$.

The membership functions are not of the same size since it is desirable to have finer distinction between grades in the lower and higher end of the influence scale. As an example, three experts proposed different linguistic weights for the interconnection $\boldsymbol{w}_{j i}$ from concept $\boldsymbol{C}_{j}$ to concept $\boldsymbol{C}_{i}: 1$ ) positively high; 2) positively very high; and 3 ) positively very-very high. The three suggested linguistics are integrated using a sum combination method and then the defuzzification method of center of gravity $(\mathrm{CoG})$ is used to produce a weight $\boldsymbol{w}_{j i}=0$, 77 in the interval $[-1,1]$. This approach has the advantage that experts do not have to assign numerical causality weights but to describe the degree of causality among concepts. The same semantic rule and term set can be used to define the coefficients $\boldsymbol{k}_{1}$ and $\boldsymbol{k}_{2}$.

A similar methodology can be used to assign values to concepts. The group of experts is also asked to describe the measurement of each concept using once again linguistic notions. Measurement of a concept is also interpreted as a linguistic variable with values in the interval $[-1,1]$. Its term set $\boldsymbol{T}$ (Measurement) $=\boldsymbol{T}$ (Influence). A new semantic rule $\boldsymbol{M}_{2}$ (analogous to $\boldsymbol{M}$ ) is also defined and these terms are characterized by the fuzzy sets whose membership functions $\boldsymbol{\mu}_{2}$ are analogous to membership functions $\boldsymbol{\mu}$.

2) Learning Algorithms: The proposed methodology tool assumes that the weight matrix is built and maintained based on expert input. Essentially, experts provide individual matrices, which are combined using a simple unsupervised inference law. The proposed methodology assumes that skeleton FCMs remain stable enough during the whole PDC activities. Skeleton FCMs change only when the underlying business model changes. Similarly, business cases (scenarios) change only if

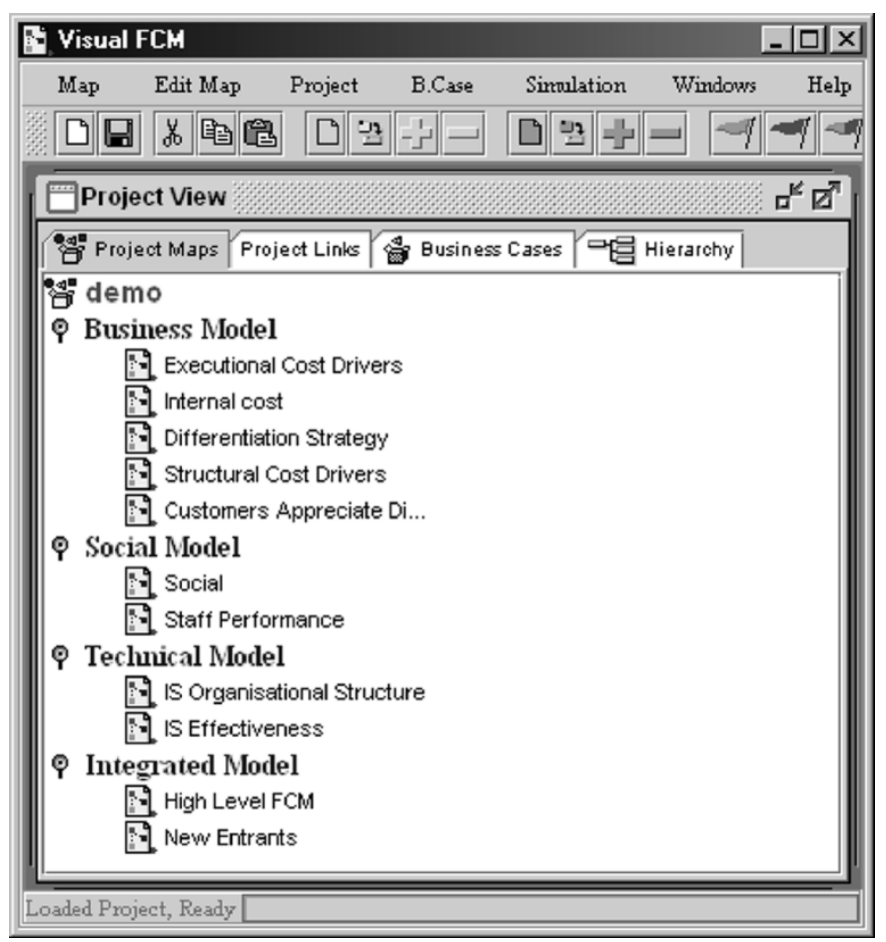

Fig. 7. Map categories.

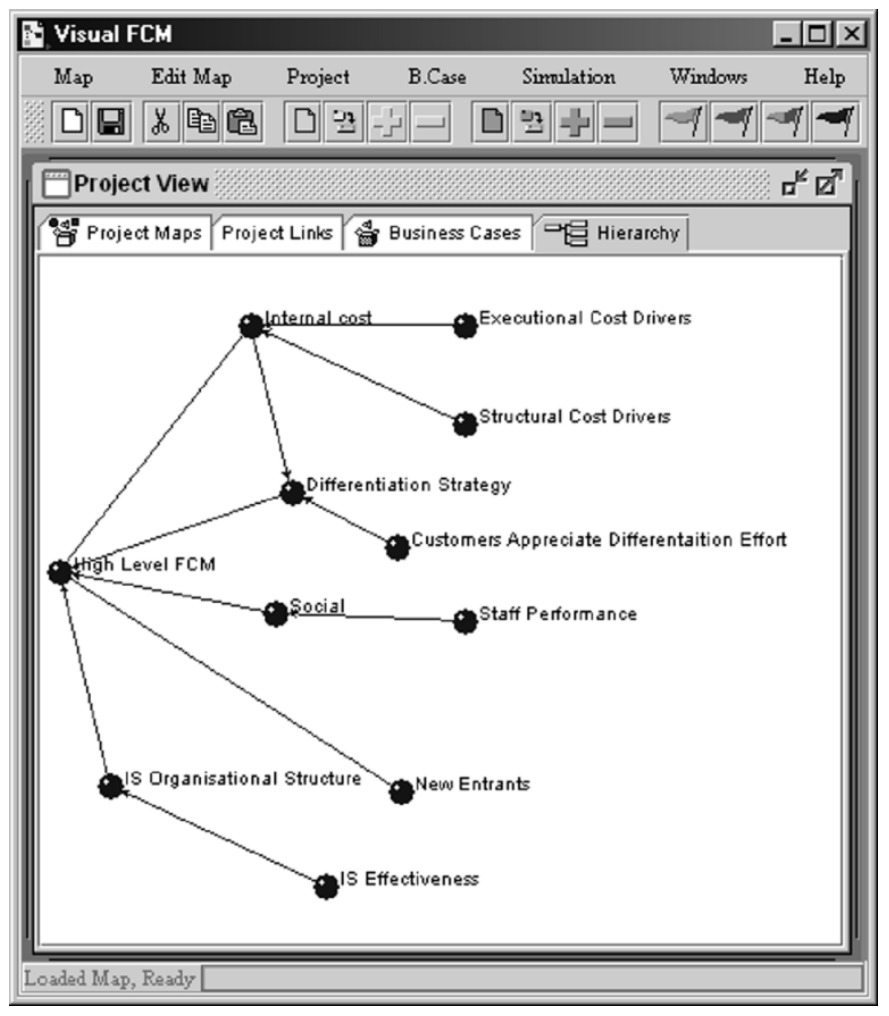

Fig. 8. Sample FCM hierarchy.

the underlying process reengineering hypotheses change. In practice, business cases may not change significantly (if any at all) if every distinct reengineering hypothesis forms the basis for a new distinct business case. These assumptions of relatively stable FCM structures couple with the design choice 


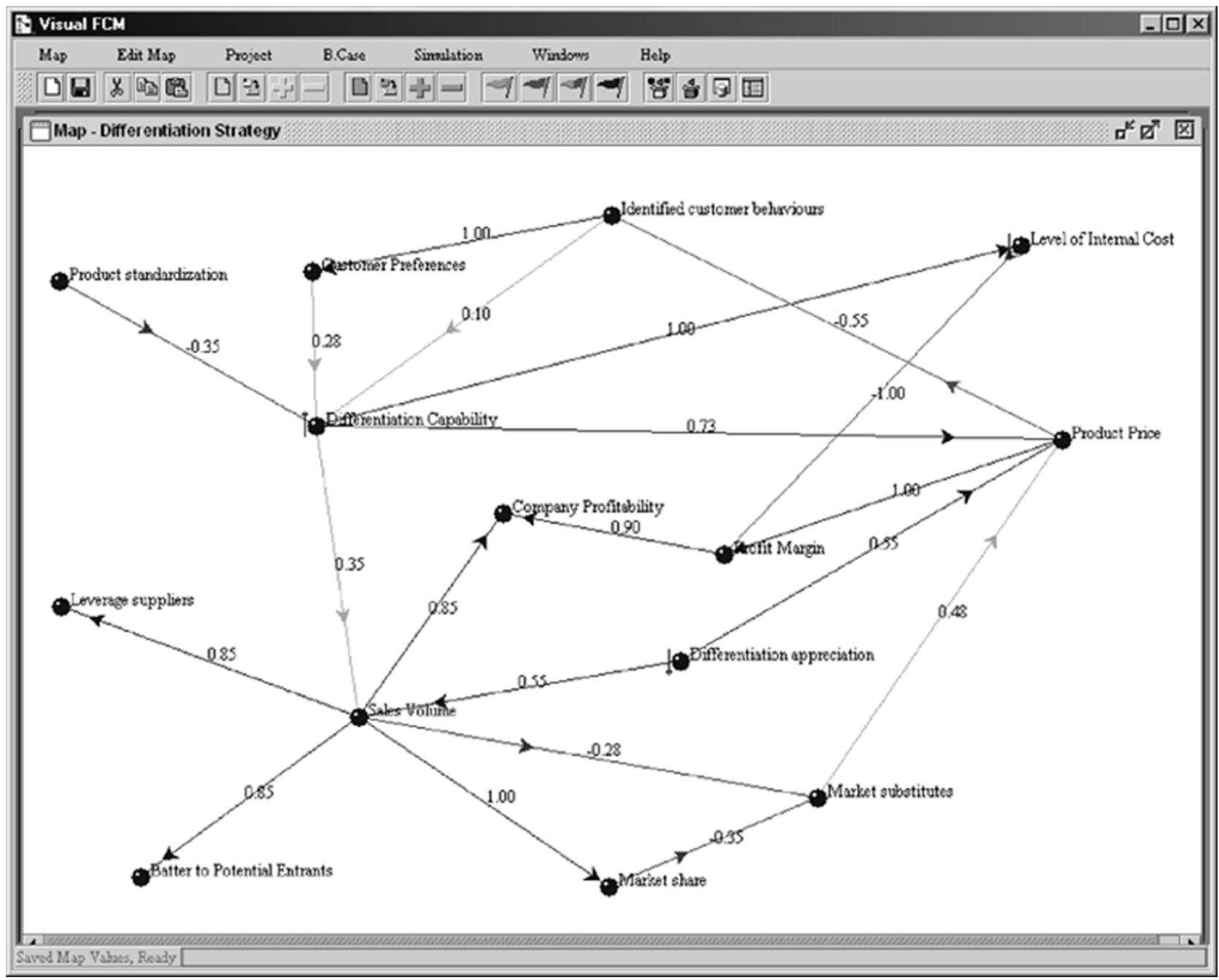

Fig. 9. Differentiation strategy FCM.

of having experts assign linguistics variables, since such assignments ultimately assimilate past experience, informal training sessions, brainstorming, best practices, current enterprise status analysis, etc., which usually do not change fundamentally during the course of the same BPR exercise. To extend this syllogism further, variable assignments may be modified (when necessary) by the application of semantic rules $\boldsymbol{M}$ and $\boldsymbol{M}_{2}$.

However, recent research activities indicate that this unsupervised "training" operation can be replaced by the automated creation (and modification) of FCMs from raw data. Kosko [33] discussed that traditional learning laws (e.g., differential hebbian learning law) can provide conditionally stable dynamic systems able to express the hidden patterns of adaptive causal networks. Other variations of automated creation/modification techniques include balanced differential learning, learning rules with generalized hebbian synapses [3], pseudo outer-product learning algorithms to reduce the work in identifying fuzzy rules [56], generalized hebbian rules for activity-dependent modifications [28], etc. The integration of such a learning inference technique currently falls out of the scope of the proposed mechanism, however, it is considered as a future research direction.

\section{PResentation of Generic FCMs}

\section{A. FCM Hierarchies}

This paper introduces generic maps that can supplement the strategic planning and business analysis phases of typical redesign projects. This research team uses the Quanta application tool, a robust visual implementation of FCMs. The implementation of Quanta has been funded by the ESPRIT E.U. programme. The proposed FCMs store concepts under four different map categories (Fig. 7), namely

- Business category: all concepts relating to core business activities;

- HR category: all human resources related concepts.

- Infrastructure category: all infrastructure related concepts with emphasis on technology infrastructure;

- Integrated category: essentially concepts which fall under more than one of the above three categories, also, top-most concepts (e.g., a concept $\boldsymbol{C}_{i}$ with no backward causality such that $\left.\forall \mathbf{j}: \boldsymbol{w}_{j i}=\mathbf{0}\right)$.

This categorization is compatible with the "process view" of the enterprise during BPR exercises, in contrast to the "organizational view" adopted by [35]. The hierarchical decomposition 


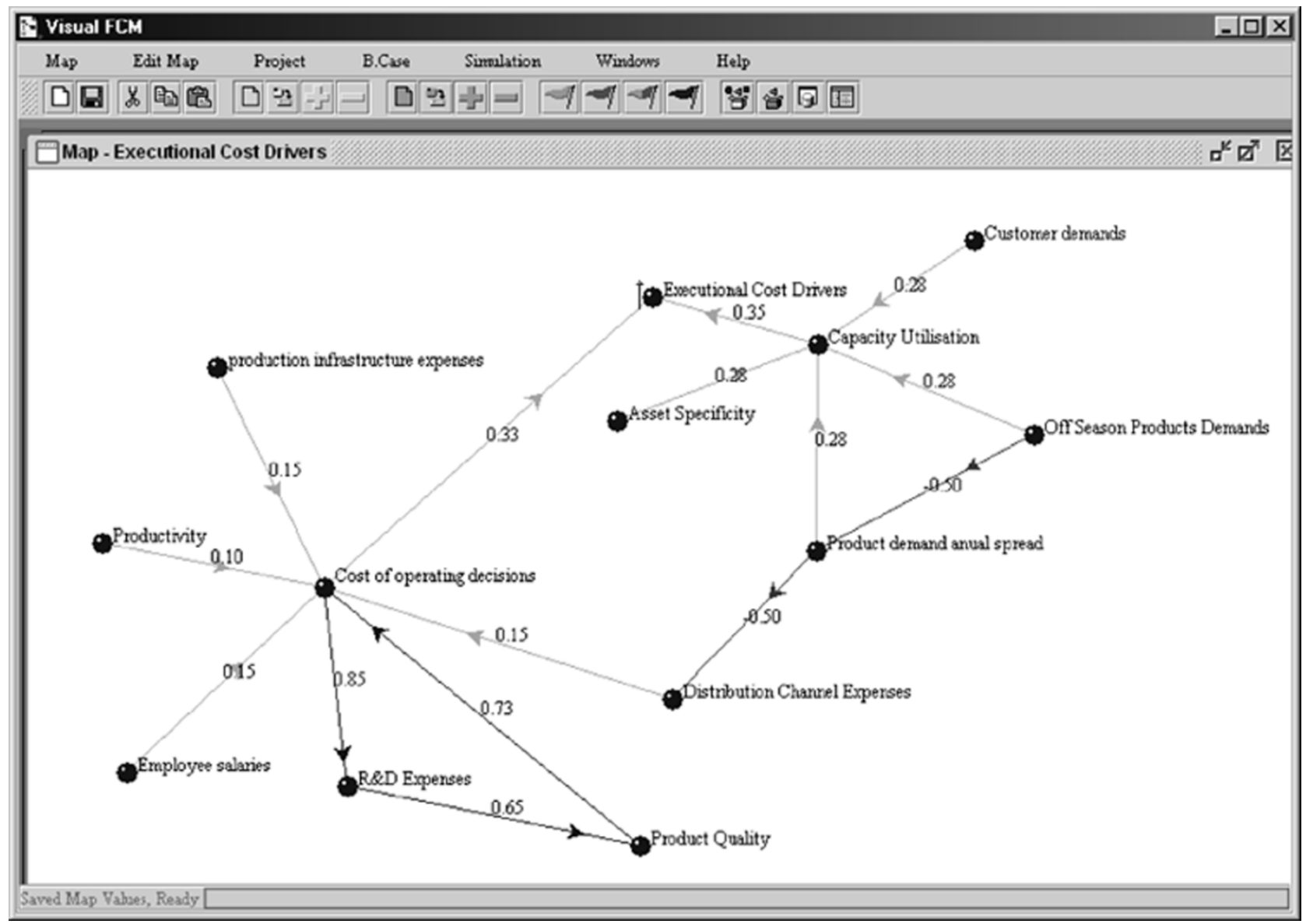

Fig. 10. Execution cost FCM.

of metrics generates a set of dynamically interconnected hierarchical maps. Each map analyzes further the relationships among concepts at the same hierarchical level. Fig. 8 presents such a sample map hierarchy, which also serves as the FCM overview.

Currently, the mechanism integrates more than 250 concepts forming a hierarchy of more than ten maps. The dynamic interface of the mechanism allows the redesigner to utilize a subset of these 250 concepts by setting the value of the redundant concepts and/or the value of the associated weights to zero.

The model can portray both the business model and the BPR exercise following either a holistic or a scalable approach. This is analogous to seeing BPR either as a single, "big bang" event or as an ongoing process of targeting successive BPR subprojects to selected subprocesses. The proposed mechanism can accommodate both approaches. Essentially, the implementation can decompose concepts to their constituent parts (subconcepts) on demand and let the user reason about lower level hierarchies of FCM before it passes values to the higher level hierarchies. The proposed mechanism also allows the user to specify the degree of FCM decomposition during the map traversal. Instead of waiting for a lower level FCM to traverse its nodes and pass its value to higher level map hierarchies, the user may assign directly an external value to nodes which link hierarchies. In practice, the simulation is carried out as if there are no links with other FCMs.

The following sections present sample skeleton maps for all four categories and provides the rationale behind each map.

\section{B. Business Metrics}

The mechanism proposes four different maps each consisting of generic business metrics as follows.

- The "differentiation strategy" map (Fig. 9) reasons on the impact of the process change to the competitive strategic identity of the enterprise.

- The "internal cost" map summarizes concepts (metrics), which affect the overall cost of delivering products (or services) to the clients of the enterprise (e.g., value chain optimization, staff performance, execution cost, etc.).

- The "execution cost" map (Fig. 10) interconnects concepts that influence the production cost of the enterprise.

- The "structural cost" map reasons on the impact of changes like outsourcing, product orientation, economies of scale, etc., to the business model.

- The "customers' appreciation" map reasons on the impact of customer satisfaction in the differentiation effort of the enterprise.

Concepts denoted as " $\downarrow$ " expand further to lower level maps.

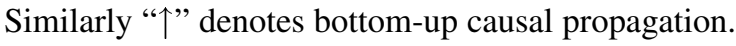

\section{Human Resources (HR) Metrics}

The mechanism proposes two different maps each consisting of generic HR category metrics as follows.

- The "staff performance" map (Fig. 11) summarizes concepts (metrics), which affect the overall performance of 


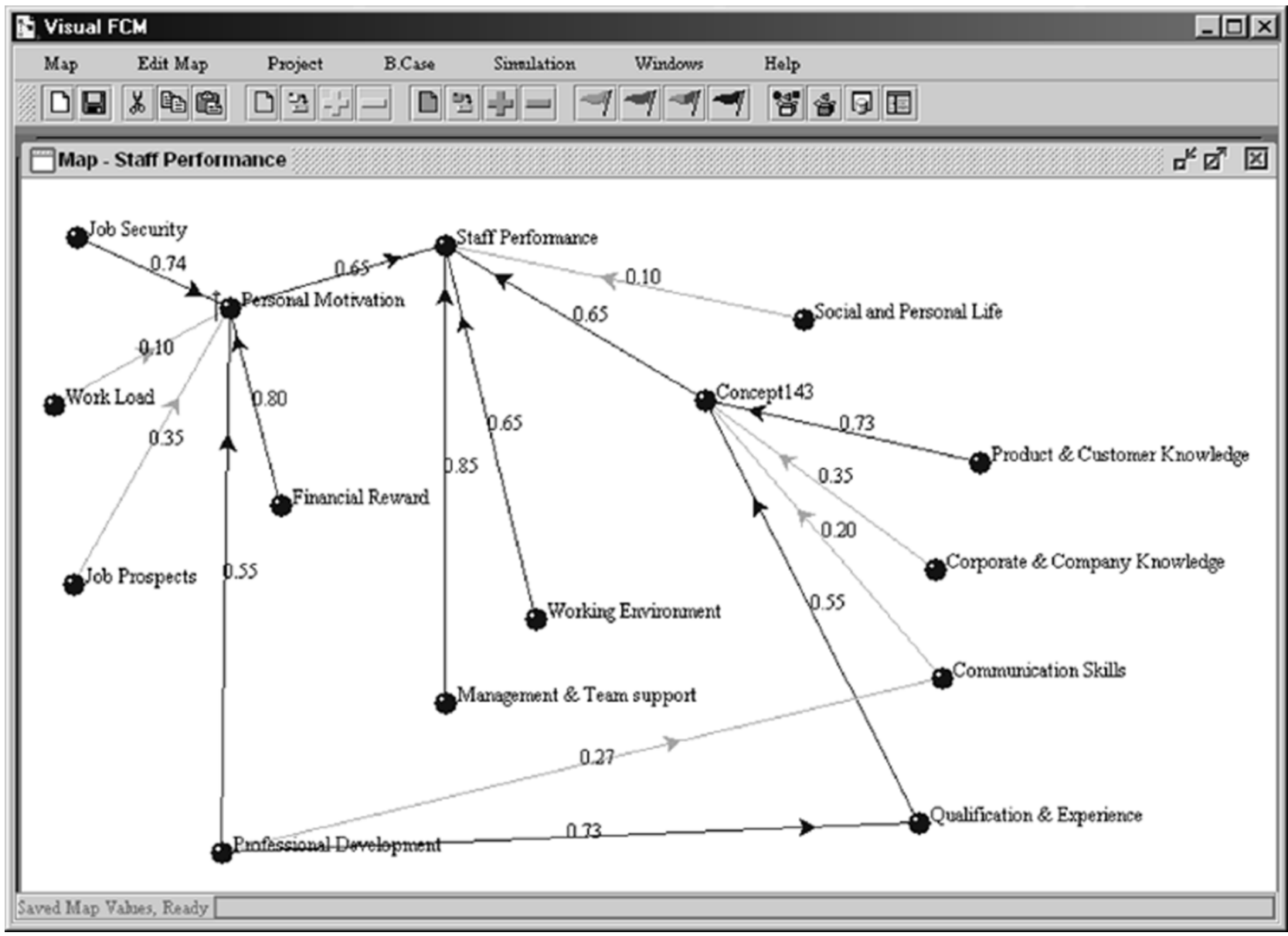

Fig. 11. Staff performance FCM.

the human resources (e.g., motivation, carrier prospects, financial rewards, work load, etc.)

- The "social" map (Fig. 12) supports reasoning of the impact of knowledge management, training, and employee satisfaction to the business model.

\section{Infrastructure Metrics}

Metrics of the infrastructure category generate two maps, each consisting of interconnected business concepts as follows.

- The "information systems (IS) organizational structure" map (Fig. 13) supports reasoning of the impact of the centralization/decentralization of the information systems of the enterprise to the overall business model.

- The "Information systems effectiveness" map supports reasoning of the changes in the technology infrastructure of the enterprise.

\section{E. Integrated Metrics}

The proposed integrated category consists of two different maps as follows.

- The "high-level" map (Fig. 14) essentially relates concepts, which fall under more than one of the other three categories, as well as top-most concepts.
- The "new entrants" map supports reasoning of the impact of business model adjustments to meet the product standards (e.g., quality, differentiated products, price policies, etc.) set by new competitors.

All the above-mentioned maps form a generic domain of FCMs. This domain serves as the basis of the proposed approach and can be modified to comply with the requirements of specific BPR projects. For example, further maps in the technical category could relate concepts that measure the performance of the production cycle [20], [65].

\section{PRELIMINARY EXPERIMENTS}

\section{A. Nature of the Experiments}

Two experiments were conducted by utilizing metrics from actual (though random) BPR exercises in two major financial sector enterprises. For each experiment, a team of experts was engaged to:

- provide linguistic variables for the causal weights, the concept values and the coefficients values to let the FCM algorithm reason about the impact of potential change initiatives;

- provide their independent expert estimates (using similar linguistic variables) of the impact of the redesign choices to specific performance metrics. 


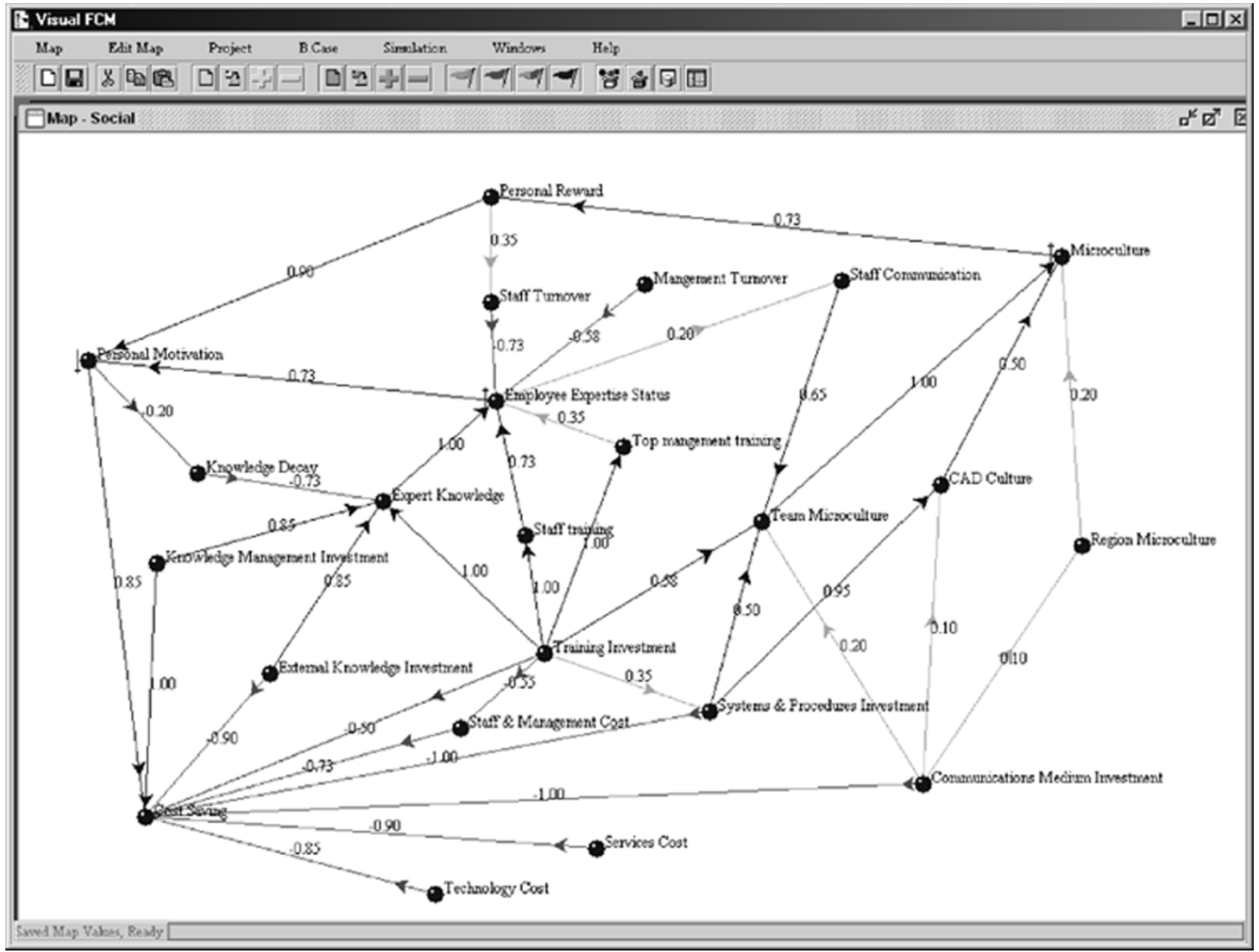

Fig. 12. Social FCM.

For both cases, the Quanta tool iterated a subset of approximately 120 concepts spread over eight sample hierarchical maps in order to calculate their equilibrium values. Both cases run on a typical business PC with a $2.4 \mathrm{GHz}$ Pentium processor and 512-MB RAM. As far as the number of iterations is concerned, lower level maps iterated ten times on average. The average number of iterations increased to 25 for middle and upper level maps. Only the top most map increased the average number of iterations to approximately 180 (depending on the initial concept and weight values) due to the volume of map links. In practice, the actual process time was negligible on such a typical PC.

Fig. 15 compares the impact of process changes with specific performance metrics as estimated by the FCM mechanism and the team of experts, respectively, for the first BPR exercise.

The majority of the selected metrics cascade to several constituent metrics. This selection allows the mechanism to express its reasoning capabilities by traversing complicated concept interrelations spreading over different maps and hierarchies. Figs. 6-11 presented sample FCMs and weight values for the first experiment generated by the application of the semantic rule $\boldsymbol{M}$ (as described in Section III-D) over the linguistic variables provided by the team of experts. The initial concept values were generated by the application of the semantic rule $\boldsymbol{M}_{2}$ (as described in Section III-D) over the linguistic variables provided by the team of experts. The FCM mechanism calculated the value of affected concepts based on the initial weight and concept value.

Similarly, Fig. 16 shows the impact of process changes to specific performance metrics as estimated by the FCM mechanism and the team of experts, respectively, for the second BPR exercise.

\section{B. Discussion}

Various aspects of the proposed modeling mechanism are now commented on. As far as the theoretical value is concerned, the proposed mechanism extends previous research attempts (as discussed in Section II-B) by the following:

- allowing fuzzy node and weight definitions in the cognitive maps;

- introducing a specific interpretation mechanism of linguistic variables to fuzzy sets;

- proposing an updated FCM algorithm to suit better the BPR domain;

- introducing the notion of interconnected performance hierarchies;

- supporting node linking to establish map hierarchies and dynamic map selection during simulation;

- concentrating on the actual BPR activity and its impact on the business model; 


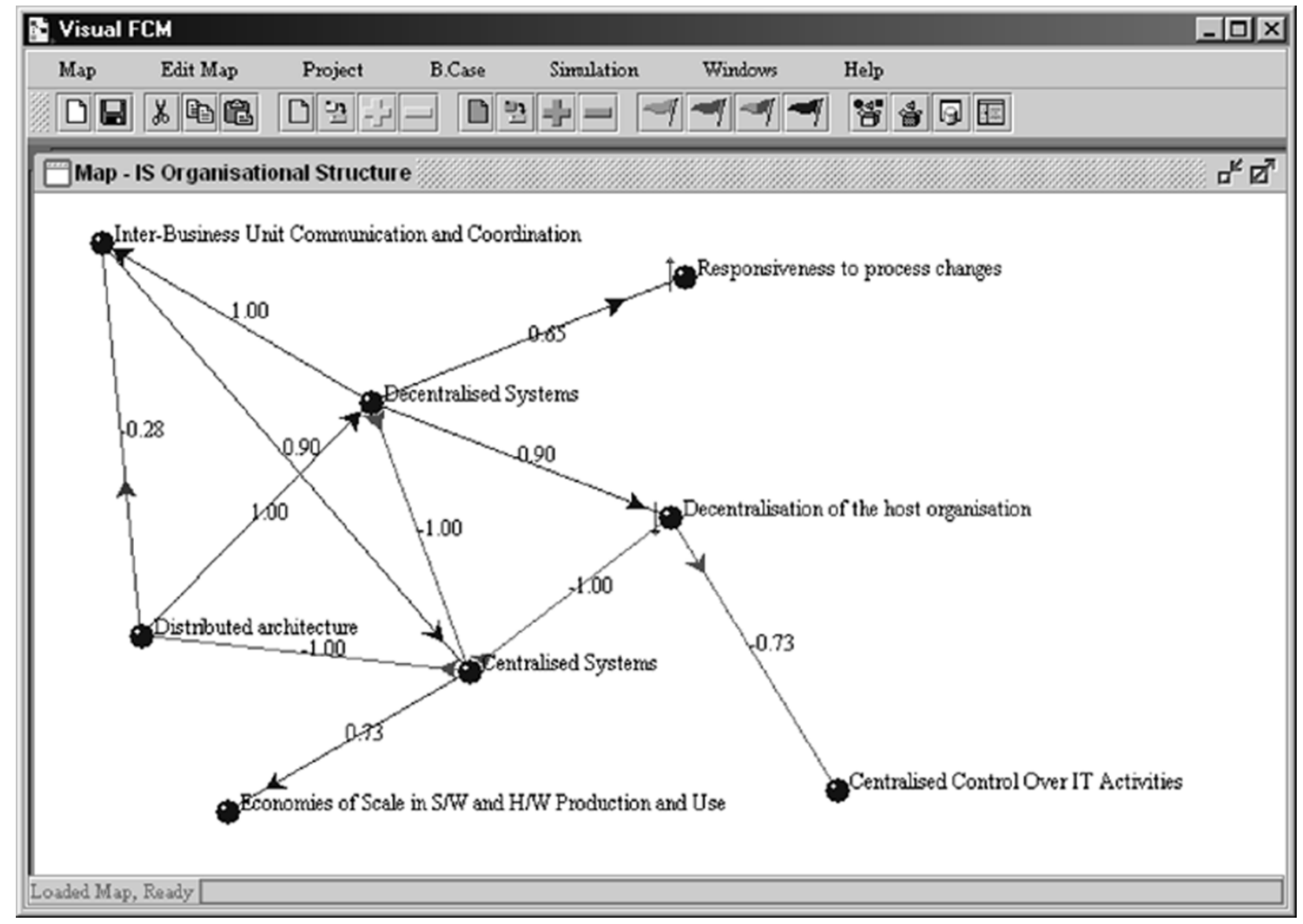

Fig. 13. IS organizational structure.

- allowing dynamic map decomposition and reconfiguration;

- integrating three modes of FCM simulation, namely, bivalent (with a crisp activation set $\{\mathbf{0 , 1}\}$ ), trivalent (also with a crisp activation set $\{-\mathbf{1}, \mathbf{0}, \mathbf{1}\}$ ), and linear (with an activation set in the fuzzy interval $[-1, \ldots, 1]$ );

- supporting the user with appropriate interface windows when loops, cycles, and node conflicts are identified.

As far as the practical value of the proposed mechanism is concerned:

- When compared with the expert estimates, the mechanism does not provide fundamentally different "diagnosis." On the contrary, it provides reasonably good approximations of the impact of redesign activities.

- In comparison to the expert estimates, the proposed mechanism tends to overestimate the effect of process change to shallow concepts (metrics) or concepts with few causal dependencies.

- In comparison to the expert estimates, the proposed mechanism tends to underestimate slightly the impact of process change to concepts (metrics), which have several constituent subconcepts or concepts, which have several hierarchical dependencies. This conservatism, however, does not reduce the effectiveness of the proposed mechanism. It simply indicates that when several complex performance factors are involved, it may be safer to assume a conservative performance improvement scenario.
- It provides a uniform behavior regardless of the degree of process change. The first experiment (see Fig. 15) involved targeted process improvement, while the second experiment (see Fig. 16) involved radical process change.

- The justification of the "diagnosis" (essentially the metrics decomposition) proved extremely helpful in comprehending the sequence of complex concept interactions (essentially the performance roadmap).

- The concept-based approach did not restrict the interpretation of the estimated impact. The fuzzy interpretation of concept and weight values served as indications rather than precise arithmetic calculations.

- The hierarchical (or partial) traversal of performance metrics improved the distributed monitoring of change activities throughout different hierarchical levels of the enterprise and stipulated targeted communication of the associated performance impact (e.g., partial impact of partial process change on intermediate metrics).

- The realism of impact estimation depended on the number of concepts and weights, as well as on the estimation of their fuzzy characteristics (weights values, input values, coefficients, etc.). However, the complexity and the length of the concept domain did not discourage the maintenance of the mechanism. Irrelevant and/or unnecessary maps could be isolated on demand to reduce the reasoning effort. 


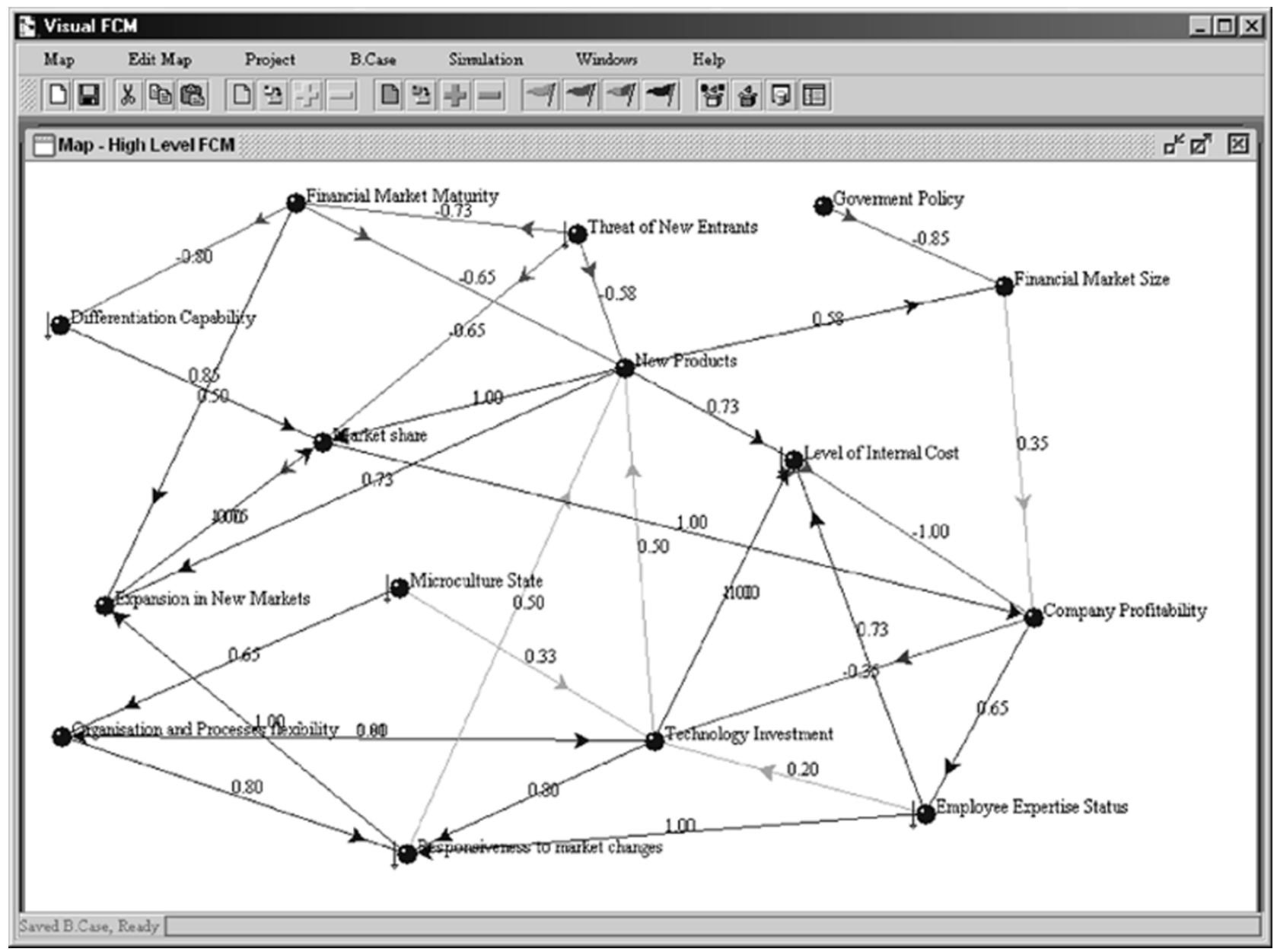

Fig. 14. High level FCM.

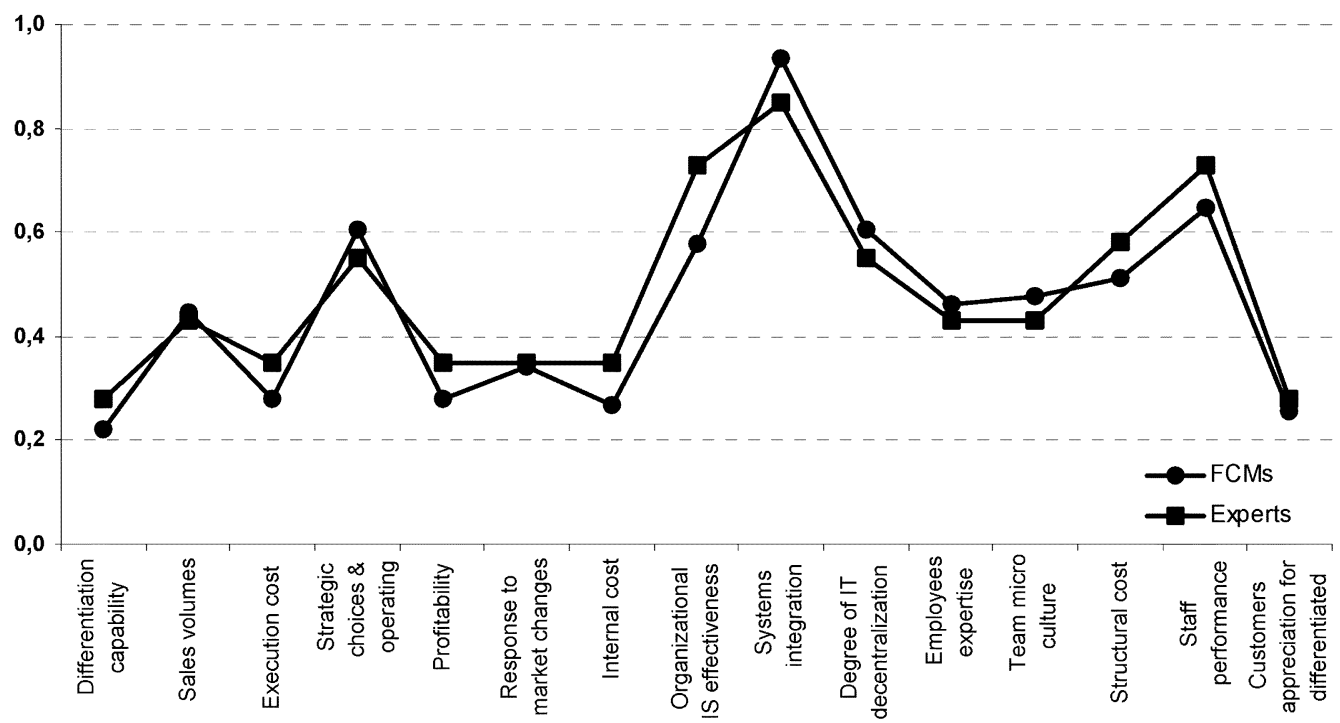

Fig. 15. Metrics estimates provided by the experts and the FCM mechanism for the first experiment.

Having established the theoretical and practical value of the proposed mechanism, it is useful to discuss also the added value of incorporating such a mechanism into BPR exercises. It is the belief of this paper that the resulting tool provides real value 


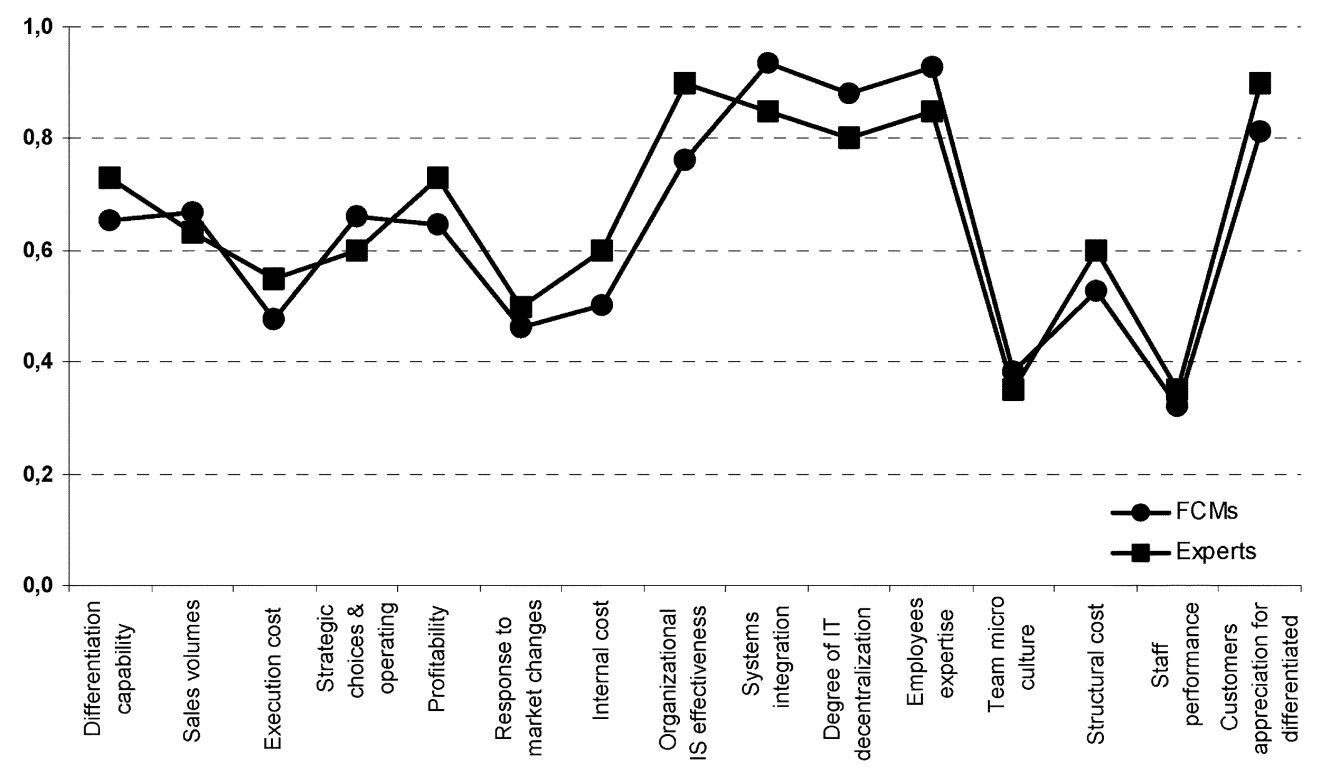

Fig. 16. Metrics estimates provided by the experts and the FCM mechanism for the second experiment.

to the principle beneficiaries and stakeholders of PDC projects (enterprise administration, redesign leaders, etc.). For example, the following.

- The mechanism eases significantly the complexity of deriving expert decisions concerning the strategic planning and business analysis phases of typical BPR exercises. Informal experiments indicated that the time required by experts to estimate manually the extensive impact of major process changes to realistic business models could pose as a considerable overhead. On the other hand, the elapsed time for automated estimations using FCM decision support can be insignificant, once the map hierarchies have been set up.

- To extend further this syllogism, realistic BPR projects should involve continuous argument of process change options (e.g., application of best practices, alternative strategic planning scenarios, alternative process modifications, etc.) until an equilibrium solution accepted by all stakeholders has been agreed upon. Informal discussions with the principle beneficiaries and stakeholders of the two BPR projects revealed that the proposed FCM decision support can reduce significantly the overheads of impact estimation, letting the stakeholders focus on the actual BPR exercise, while exploring in depth all alternatives and controlling effectively major change initiatives.

- The proposed mechanism can also assist the post-BPR performance evaluation of the enterprise on a regular basis. FCMs may serve as a back end to performance scorecards ([4], [23], [24]) to provide holistic strategic performance evaluation and management. However, a detailed analysis of this extension falls out of the scope of this paper.

Senior managers of the two major financial sector enterprises have evaluated the usability of the proposed tool and have identified a number of benefits that can be achieved by the utilization of the proposed FCM tool as a methodology framework for process redesign and performance measurement. Detailed pre- sentations of the results from the usability evaluation fall out of the scope of the paper; however, a summary of major business benefits (as identified by senior managers) is provided to improve the autonomy of this paper.

\section{1) Shared Goals}

- Concept-driven simulation pulls individuals together by providing a shared direction and determination of process change.

- Shared BPR planning and performance measurement enables business units to realize how they fit into the overall business model of the enterprise and what is their actual contribution.

- Senior management receives valuable inputs from the business units (or the individual employees) who really comprehend the weaknesses of the current process model, as well as the opportunities for performancedriven process change.

\section{2) Shared Culture}

- All business units feel that their individual contribution is taken under consideration and provide valuable input to the whole change process.

- All business units and individuals feel confident and optimistic; they realize that they will be the ultimate beneficiaries of the BPR exercise.

- The information sharing culture supports the enterprise's competitive strategy and provides the energy to sustain this by exploiting fully the group and the individual potential.

3) Shared Learning

- The enterprise realizes a high return from its commitment to its human resources.

- There is a constant stream of improvement within the enterprise.

- The entire enterprise becomes increasingly receptive to process changes, since the benefit can be easily demonstrated to individual business units. 


\section{4) Shared Information}

- All business units and individuals have the necessary information needed to set clearly their objectives and priorities.

- Senior management can control effectively all aspects of the redesign process.

- The enterprise reacts rapidly to threats and opportunities.

- It reinforces trust and respect throughout the enterprise.

Summarizing, experiments showed that FCM-based ex ante reasoning of the impact of process model changes (actual or hypothetical) to the status of performance metrics can be effective and realistic. This is considered to be a major contribution of the proposed tool to actual BPR exercises.

\section{CONCLUSION}

This paper presented a supplement to the BPR methodology based on FCMs. The proposed decision aid supplements the strategic planning and business analysis phases of typical BPR projects by supporting "intelligent" reasoning of the anticipated ("to-be") business performance. By using FCM, the proposed mechanism drew a causal representation of business performance principles; it simulated the operational efficiency of complex process models with imprecise relationships and quantified the impact of the reengineering activities to the business model.

This paper discussed the FCM approach in putting realistic and measurable objectives in BPR projects and presented sample maps with concept causal relationships. Preliminary experiments indicated that the mechanism did not provide fundamentally different estimates than expert decisions. It provided reasonably good estimates of the impact of redesign activities to the business model, while the maintenance effort did not pose as a prohibitory factor. Moreover, the decomposition of performance metrics supported reasoning of the performance roadmap and the complex relationships that affect the overall performance of the business model.

The proposed mechanism should not be regarded only as an effective business modeling support tool. Its main purpose is to drive process change activities rather than limit itself to qualitative simulations. Moreover, the proposed mechanism should not be seen as an "one-off" decision aid. It should be a means for setting a course for continuous improvement [36].

Future research will focus on conducting further real-life experiments to test and promote the usability of the tool, but also to identify potential pitfalls. Furthermore, future research will focus on the automatic determination of appropriate fuzzy sets (e.g., utilizing pattern recognition, mass assignments, empirical data, etc.) for the representation of linguistic variables to suit each particular BPR project domain. Finally, further research will focus on implementing backward map traversal, a form of adbuctive reasoning [13]. This feature offers the functionality of determining the condition(s) $\boldsymbol{C}_{i j}$ that should hold in order to infer the desired $\boldsymbol{C}_{j}$ in the causal relationship $C_{i j} \stackrel{\mathrm{w}_{j k}}{\longrightarrow} C_{k}$. Incorporating performance integrity constraints reduces the search space and eliminates combinatory search explosion.
Backward reasoning has been tested extensively in other applications and its integration in the proposed methodology framework may prove beneficiary.

\section{ACKNOWLEDGMENT}

The authors wish to thank C. Apostolou, P. Chytas, G. Nikolakis, and M. Lazaridis for participating in the evaluation of the proposed tool.

\section{REFERENCES}

[1] M. Al-Mashari and M. Zairi, "Revisiting BPR: A holistic review of practice and development," J. Bus. Process Manage., vol. 6, no. 1, pp. 10-42, 1999.

[2] R. Axelrod, Structure of Decision: The Cognitive Maps of Political Elites. Princeton, NJ: Princeton Univ. Press, 1976.

[3] F. Botelho and J. E. Jamison, "A learning rule with generalized Hebbian synapses," J. Math. Anal. Applicat., vol. 273, pp. 29-547, 2002.

[4] M. Bourne, J. Mills, M. Wilcox, A. Neely, and K. Platts, "Designing, implementing and updating performance measurement systems," Int. J. Qual. Rel. Prod. Manage., vol. 20, pp. 754-771, 2000.

[5] T. F. Burgess, "Modeling quality-cost dynamics," Int. J. Qual. Rel. Manage., vol. 13, no. 3, pp. 8-26, 1996.

[6] - "Modeling the impact reengineering with system dynamics," Int. J. Qual. Rel. Prod. Manage., vol. 18, no. 9/10, pp. 950-963, 1998.

[7] C. Carlsson and E. Turban, "DSS: Directions for the next decade," J. Decision Support Syst., vol. 33, no. 1, pp. 105-110, 2002.

[8] J. P. Craiger, D. F. Goodman, R. J. Weiss, and A. Butler, "Modeling organizational behavior with fuzzy cognitive maps," J. Comput. Intell. Organ., vol. 1, pp. 120-123, 1996.

[9] T. J. Crowe, P. M. Fong, T. A. Bauman, and J. L. Zayas-Castro, "Quantitative risk level estimation of business process reengineering efforts," Bus. Process Manage. J., vol. 8, no. 5, pp. 490-511, 2002.

[10] T. H. Davenport, Process Innovation: Reengineering Work Through Information Technology. Boston, MA: Harvard Business School, 1993.

[11] J. A. Dickerson and B. Kosko, "Virtual worlds as fuzzy cognitive maps," in Fuzzy Engineering, B. Kosko, Ed. Upper Saddle River, NJ: Prentice-Hall, 1997.

[12] J. Diffenbach, "Influense diagrams for complex strategic issues," Strategic Manag. J., vol. 3, pp. 133-146, 1982.

[13] P. A. Flach and A. Kakas, "Abduction and induction in AI: Report of the IJCAI97 workshop," Logic J. Interest Group on Pure Appl. Logic, vol. 6, no. 4, pp. 651-656, 1998.

[14] V. Georgopoulos, G. Malandraki, and C. Stylios, "A fuzzy cognitive map approach to differential diagnosis of specific language impairment," $J$. Artif. Intell. Med., vol. 679, pp. 1-18, 2002.

[15] M. Hagiwara, "Extended fuzzy cognitive maps," in Proc. 1st IEEE Int. Conf. Fuzzy Systems, New York, 1992, pp. 795-801.

[16] M. Hammer and J. Champy, Reengineering the Corporation: A Manifesto for Business Revolution. New York: Harper Collins, 1993.

[17] P. Harmon and D. King, Expert Systems: Artificial Intelligence in Business. New York: Wiley, 1985.

[18] H. J. Harrington, Business Process Improvement. New York: McGrawHill, 1991.

[19] T. Hong and I. Han, "Knowledge based data mining of news information of the internet using cognitive maps and neural networks," J. Expert Syst. Applicat., vol. 23, no. 1, pp. 1-8, 2002.

[20] Z. Irani, V. Hlupic, L. P. Baldwin, and P. E. D. Love, "Re-engineering manufacturing processes through simulation modeling," J. Logistics Inform. Manage., vol. 13, no. 1, pp. 7-13, 2002.

[21] R. J. Johnson and R. O. Briggs, "A model of cognitive information retrieval for ill-structured managerial problems and its benefits for knowledge acquisition," in Proc. 27th Annu. Hawaii Int. Conf. System Sciences, Wailea, HI, 1994, pp. 191-200.

[22] R. T. Jones and C. Ryan, "Matching process choice and uncertainty: Modeling quality management," J. Bus. Process Manage., vol. 8, no. 2, pp. 161-168, 2002.

[23] R. S. Kaplan and D. P. Norton, "Using the balanced scorecard as a strategic management system," in Harv. Bus. Rev., Jan./Feb. 1996, pp. 75-85.

[24] _ "Leading change with the balanced scorecard," Financial Executive, vol. 17, pp. 64-66, 2001.

[25] D. Kardaras and B. Karakostas, "The use of fuzzy cognitive maps to simulate the information systems strategic planning process," J. Inform. Software Technol., vol. 41, no. 1, pp. 197-210, 1999.

[26] A. Kaufmann, Introduction to the Theory of of Fuzzy Subsets, Fundamental Theoretical Elements. New York: Academic, 1975. 
[27] H. S. Kim and K. C. Lee, "Fuzzy implications of fuzzy cognitive map with emphasis on fuzzy causal relationship and fuzzy partially causal relationship," Fuzzy Sets and Syst., vol. 97, pp. 303-313, 1998.

[28] T. Kitajima and K. Hara, "A generalized Hebbian rule for activity-dependent synaptic modifications," J. Neural Networks, vol. 13, pp. 445-454, 2000.

[29] J. C. Klein and D. F. Cooper, "Cognitive maps of decision makers in a complex game," J. Oper. Res. Soc., vol. 33, pp. 63-71, 1982.

[30] B. Kosko, "Differential Hebbian learning," in Proc. AIP Conf. 151, 1986, pp. $265-270$.

[31] - "Fuzzy cognitive maps," J. Man-Machine Studies, vol. 24, pp. $65-75,1986$.

[32] — Neural Networks and Fuzzy Systems. Englewood Cliffs, NJ: Prentice-Hall, 1991

[33] —_ "Hidden patterns in combined and adaptive knowledge networks," Int. J. Approximate Reasoning, vol. 2, pp. 377-393, 1998.

[34] M. E. Kuwaiti and J. M. Kay, "The role of performance measurement in business process re-engineering," Int. J. Oper. Prod. Manage., vol. 20, no. 12, pp. 1411-1426, 2000

[35] K. Y. Kwahk and Y. G. Kim, "Supporting business process redesign using cognitive maps," Decision Support Syst., vol. 25, no. 2, pp. $155-178,1999$.

[36] M. Langbert and H. Friedman, "Continuous improvement in the history of human resource management," J. Manage. Decision, vol. 40, no. 8, pp. $782-787,2002$

[37] K. C. Lee and H. S. Kim, "A fuzzy cognitive map-based bi-directional inference mechanism: An application to stock investment analysis," $J$. Intell. Syst. Accounting Finance Manage., vol. 6, no. 1, pp. 41-57, 1997.

[38] — - "Fuzzy implications of fuzzy cognitive map with emphasis on fuzzy causal relationship and fuzzy partially causal relationship," $J$. Fuzzy Sets Syst., vol. 3, pp. 303-313, 1998.

[39] K. C. Lee, J. S. Kim, N. H. Chung, and S. J. Kwon, "Fuzzy cognitive map approach to web-mining inference amplification," J. Expert Syst. Applicat., vol. 22, pp. 197-211, 2002.

[40] K. C. Lee and O. B. Kwon, "A strategic planning simulation based on cognitive map knowledge and differential game," J. Simulation, vol. 7, no. 5, pp. 316-327, 1998

[41] X. Li and F. Lara-Rosano, "Adaptive fuzzy petri nets for dynamic knowledge representation and inference," J. Expert Syst. Applicat., vol. 19, no. 3 , pp. 235-241, 2000

[42] F. R. Lin, M. C. Yang, and Y. H. Pai, "A generic structure for business process modeling," Bus. Process Manage. J., vol. 8, no. 1, pp. 19-41, 2002.

[43] Z. Q. Liu, Fuzzy Cognitive Maps: Analysis and Extension. Tokyo, Japan: Springer, 2000.

[44] Z. Q. Liu and R. Satur, "Contexual fuzzy cognitive map for decision support in geographical information systems," Trans. Fuzzy Systems, vol. 7, pp. 495-507, 1999.

[45] C. M. MacNeil, "Line managers: Facilitators of knowledge sharing in teams," J. Employee Relations, vol. 25, no. 3, pp. 294-307, 2003.

[46] K. Metaxiotis, J. Psarras, and E. Samouilidis, "Integrating fuzzy logic into decision support systems: Current research and future prospects," $J$. Inform. Manage. Comput. Security, vol. 11, no. 2, pp. 53-59, 2003.

[47] D. Morris and J. Brandon, Re-Engineering Your Business. New York: McGraw-Hill, 1993.

[48] M. A. Murray, H. R. Priesmeyer, L. F. Sharp, R. Jensen, and G. Jensen, "Nonlinearity as a tool for business process reengineering," Bus. Process Manage. J., vol. 6, no. 4, pp. 304-313, 2000.

[49] T. D. Ndouse and T. Okuda, "Computational intelligence for distributed fault management in networks using fuzzy cognitive maps," in Proc. IEEE Int. Conf. Communication Converging Technology Tomorrow's Application, New York, 1996, pp. 249-259.

[50] J. B. Noh, K. C. L. Lee, J. K. Kim, J. K. Lee, and S. H. Kim, "A casebased reasoning approach to cognitive map driven -driven tacit knowledge management," J. Expert Syst. Applicat., vol. 19, pp. 249-259, 2000.

[51] M. A. Ould, "Process modeling with RADS," J. IOPener, vol. 1, no. 5, pp. 56-69, 1992.

[52] M. Parenthoen, P. Reignier, and J. Tisseau, "Put fuzzy cognitive mapsto work in virtual worlds," in Proc. Fuzz-IEEE'01, Melbourne, Australia, 2001, pp. 115-121.

[53] C. E. Pelaez and J. B. Bowles, "Applying fuzzy cognitive maps knowledge representation to failure modes effect analysis" in Proc. IEEE Annu. Reliability Maintainability Symp., New York, 1995, pp. 450-456.

[54] K. Perusich, "Fuzzy cognitive maps for political analysis," in Proc. Int. Symp. Technology and Society. Technical Expertise and Public Decisions, New York, 1996, pp. 369-373.

[55] D. P. Petrozzo and J. C. Stepper, Successful Reengineering. New York: Van-Nostrand Reinhold, 1994.
[56] C. Quek and R. W. Zhou, "The POP learning algorithms: Reducing work in identifying fyzzu rules," J. Neural Networks, vol. 14, pp. 1431-1445, 2001.

[57] A. Ramaprasad and E. Poon, "A computerised interactive technique for mapping influense diagrams (MIND)," Strategic Manage. J., vol. 6, no. 4, pp. 377-392, 1985.

[58] M. Schneider, E. Schnaider, A. Kandel, and G. Chew, "Constructing fuzzy cognitive maps," in Proc. IEEE Conf. Fuzzy Systems, New York, 1995, pp. 2281-2288.

[59] J. P. Shim, M. Warkentin, J. F. Courtney, D. J. Power, R. Sharda, and C. Carlsson, "Past, present, and future of decision support technology," $J$ Decision Support Syst., vol. 33, no. 1, pp. 111-126, 2002.

[60] P. C. Silva, "New forms of combinated matrices of fuzzy cognitive maps," in IEEE Int. Conf. Neural Networks, New York, 1995, pp. 71-776.

[61] R. H. Sprague and H. J. Watson, Decision Support Systems: Putting Theory into Practice. Englewood Cliffs, NJ: Prentice-Hall, 1986.

[62] C. D. Stylios, V. C. Georgopoulos, and P. P. Groumpos, "Introducing the theory of fuzzy cognitive maps in distributed systems," in Proc. 12th IEEE Int. Symp. Intelligent Control, Istanbul, Turkey, 1997.

[63] M. Vakola and Y. Rezgui, "Critique of existing business process re-engineering methodologies: The development and implementation of a new methodology," J. Bus. Process Manage., vol. 6, no. 3, pp. 238-250, 2000

[64] G. Valiris and M. Glykas, "Critical review of existing BPR methodologies. The need for a holistic approach," J. Bus. Process Manage., vol. 5, no. 1, pp. 65-86, 1999.

[65] — "A case study on reengineering manufacturing processes and structures," J. Knowl. Process Manage., vol. 7, no. 1, pp. 20-28, 2000.

[66] G. Xirogiannis, J. Stefanou, and M. Glykas, "A fuzzy cognitive map approach to support urban design," J. Expert Syst. Applicat., vol. 26, no. 2, pp. $257-268,2004$

[67] L. A. Zadeh, "Fuzzy sets," J. Inform. Control, vol. 8, pp. 338-353, 1965.

[68] W. R. Zhang, W. Wang, and R. S. King, "A-pool: An agent-oriented open system for distributed decision process modeling," J. Org. Comput., vol 4, no. 2, pp. 127-154, 1994

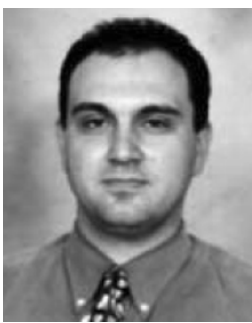

George Xirogiannis received the B.Sc. degree in mathematics from the University of Ioannina, Ioannina, Greece, in 1993, the M.Sc. degree in artificial intelligence from the University of Bristol, Bristol, U.K., in 1994, and the Ph.D. degree in computer science from Heriot-Watt University, Edinburgh, U.K., in 1998.

His $\mathrm{Ph} . \mathrm{D}$. dissertation focused on the automated parallelization of logic programming on distributed heterogeneous multiprocessors. Currently, he is a Lecturer with the Department of Financial and Management Engineering, University of the Aegean, Chios, Greece. He has obtained extensive management consulting experience as a Manager at the management consulting section of PricewaterhouseCoopers. His research interests have progressed from knowledge-based systems and distributed logic programming to computational intelligence in business analysis and modeling.

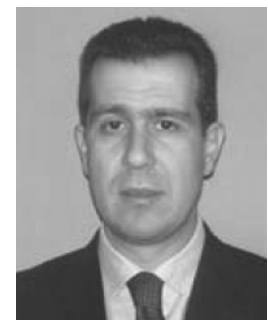

Michael Glykas received the Ph.D. degree in business process reengineering and emerging technologies from Cambridge University, Cambridge, U.K., in 1992

Since 1992, he has been a Visiting Professor at Cambridge University. Currently, he is an Assistant Professor with the Department of Financial and Management Engineering, University of the Aegean, Chios, Greece. He has obtained considerable professional experience in Europe working as a project director in major financial sector commercial projects involving business process reengineering and knowledge management. During the last ten years, he has also worked as a Technical Director in a number of E.U. funded research projects in the area of e-health and management of emerging technologies. 